\title{
Towards a photometric metallicity scale for open clusters ${ }^{\star}$
}

\author{
M. Netopil ${ }^{1}$ and E. Paunzen ${ }^{2,3}$ \\ ${ }^{1}$ Institute for Astrophysics, University of Vienna, Türkenschanzstraße 17, 1180 Vienna, Austria \\ e-mail: martin.netopil@univie.ac.at \\ 2 Department of Theoretical Physics and Astrophysics, Masaryk University, Kotlářská 2, 61137 Brno, Czech Republic \\ e-mail: epaunzen@physics.muni.cz \\ 3 Rozhen National Astronomical Observatory, Institute of Astronomy of the Bulgarian Academy of Sciences, PO Box 136, \\ 4700 Smolyan, Bulgaria
}

Received 3 May 2013 / Accepted 17 June 2013

\section{ABSTRACT}

Context. Open clusters are a useful tool when investigating several topics connected with stellar evolution; for example the age or distance can be more accurately determined than for field stars. However, one important parameter, the metallicity, is only known for a marginal percentage of open clusters.

Aims. We aim at a consistent set of parameters for the open clusters investigated in our photometric $\Delta a$ survey of chemically peculiar stars. Special attention is paid to expanding our knowledge of cluster metallicities and verifying their scale.

Methods. Making use of a previously developed method based on normalised evolutionary grids and photometric data, the distance, age, reddening, and metallicity of open clusters were derived. To transform photometric measurements into effective temperatures to use as input for our method, a set of temperature calibrations for the most commonly used colour indices and photometric systems was compiled.

Results. We analysed 58 open clusters in total. Our derived metallicity values were in excellent agreement with about 30 spectroscopically studied targets. The mean value of the absolute deviations was found to be $0.03 \mathrm{dex}$, with no noticeable offset or gradient. The method was also applied using recent evolutionary models based on the currently accepted lower solar abundance value $Z \sim 0.014$. No significant differences were found compared to grids using the former adopted solar value $Z=0.02$. Furthermore, some divergent photometric datasets were identified and discussed.

Conclusions. The method provides an accurate way of obtaining properly scaled metallicity values for open clusters. In light of present and future homogeneous photometric sky surveys, the sample of stellar clusters can be extended to the outskirts of the Milky Way, where spectroscopic studies are almost impossible. This will help for determining galactic metallicity gradients in more detail.

Key words. Hertzsprung-Russell and C-M diagrams - open clusters and associations: general - Galaxy: abundances stars: abundances - stars: evolution

\section{Introduction}

Open clusters are excellent astrophysical laboratories for almost all important processes connected with stellar formation and evolution. These include the mechanisms of diffusion, rotation, mass loss, pulsation, and accretion. One can study not only astrophysical processes, but also the connection between various star classes (e.g. variable stars) and the local metallicity or age. Star clusters are also immensely important tracers of the inner and outer galactic spiral arms.

One important characteristic of stellar formation, the metallicity, is poorly known for galactic open clusters. In the updated catalogue by Dias et al. (2002) [version 3.3. ${ }^{1}$, this parameter is listed for about 200 clusters, fewer than for $10 \%$ of the currenty known population. These values were derived from a wide variety of applied methods, ranging from metallicitydependent photometric indices and isochrone fitting, to low- and high-resolution spectroscopy. The usual convention for expressing metallicity is the logarithmic term $[\mathrm{Fe} / \mathrm{H}]$, which represents the abundance ratio of iron to hydrogen in the stellar atmosphere.

* Figure 7 is available in electronic form at http://www . aanda. org

1 http://www . astro.iag.usp.br/ wilton/
Carrera \& Pancino (2011) have compiled an almost complete list of clusters studied with medium- or high-resolution $(R \geqq$ $15000)$ spectroscopy. By also including recent literature, the spectroscopically investigated sample comprises about 100 targets so far. Most targets were studied only once, and the mean cluster metallicities were on average based on a maximum of three stars. These are generally red giants since they are the brightest cluster objects. However, Santos et al. (2012) find differences for white dwarfs and giant stars, mainly because of the use of improper line lists for evolved objects.

Some photometric systems also provide metallicity estimates (e.g. the Strömgren, Johnson, or DDO systems), but these also suffer from temperature range restrictions. In the work by Paunzen et al. (2010), photometric results were compiled for 188 open clusters in total. This list also shows that the majority of targets were investigated only once with data for only a few stars, and large differences were noticed when comparing various sources.

Studies based on colour-magnitude diagrams and isochrone fitting usually neglect the metallicity parameter, setting it for the sake of simplicity to a solar value for the targets (e.g. Kharchenko et al. 2005; Subramaniam et al. 2010; Glushkova et al. 2013). Pöhnl \& Paunzen (2010), on the other hand, present a method that offers a valuable approach to investigating 
metallicities using evolutionary models and photometric data of all main-sequence stars in a cluster. Nevertheless, it is necessary to investigate a larger sample of objects to verify whether this method provides properly scaled results.

\section{Method and target selection}

To investigate open cluster parameters in a consistent way, we applied the method by Pöhnl \& Paunzen (2010). They calculated differential evolutionary tracks (normalised to the zero age main-sequence, ZAMS) for a variety of metallicity/age combinations. These have to be compared with the observed Hertzsprung-Russell diagram (HRD) of open-cluster main-sequence stars. Since spectroscopically determined effective temperatures are not readily available, photometric data have to be transformed to the $T_{\text {eff }} / \log \left(L / L_{\odot}\right)$ plane. Using an iterative procedure, the cluster parameters are altered until the best final solution for all parameters is found. It is beneficial when some of the input parameters (the age, reddening, and distance) can be restricted (e.g. available HIPPARCos parallax, reddening deduced from photometry). However, incorrectly adopted starting values can be recognised during this procedure. Where targets had distances derived from Hipparcos data (van Leeuwen 2009), they were utilised as the initial parameter. Otherwise, we made use of the literature compilation of cluster parameters by Paunzen \& Netopil (2006). This had been updated with recent investigations to obtain proper mean starting values for the age, distance, and reddening of our programme clusters.

We followed the procedure by Pöhnl \& Paunzen (2010), but made use of a broader selection of photometric data by incorporating among others near-infrared (NIR) 2MASS measurements (Skrutskie et al. 2006) for some targets. Whenever possible, we derived individual reddening values for the cluster stars. For most targets, $U B V$ or Geneva photometry are available, which allowed us to determine the colour excess for O/B type stars by means of the $Q$ method (Johnson 1958) and $X / Y$ parameters (Cramer 1982), respectively. Even cooler type stars could be de-reddened with $u v b y \beta$ data and the appropriate calibrations (e.g. Napiwotzki et al. 1993).

The target open clusters originate in the photometric $\Delta a$ survey (e.g. Netopil et al. 2007), which is dedicated to the detection of chemically peculiar (CP) stars. So far, about 80 open clusters have been covered. A homogeneous set of cluster parameters is essential to investigating possible dependencies between the occurrence of $\mathrm{CP}$ objects and metallicity or age, for example. However, very young open clusters cannot be treated safely with the applied method owing to the too inconspicuous deviation from the ZAMS and to the restriction of the grids to $\log t \geq 7.2$. Open clusters with strong differential reddening, but without sufficient photometry to determine individual reddening values, were excluded as well. Nevertheless, we analysed a significant number of 58 open clusters (listed in Table 2) with the differential grid (DG) method.

This sample incorporates about 30 targets with available spectroscopic metallicity determinations. A comparison with our results thus allowed us to verify that the DG method could provide a proper metallicity scale for open clusters (see Sect. 6).

\section{Defining the temperature scale}

To apply the DG method, it is important to define a proper temperature scale for an accurate transformation of photometric data to effective temperatures. In contrast to Pöhnl \& Paunzen (2010), who mostly used $(B-V)$ data as an input, we utilised a broader selection of temperature dependent colour indices and photometric systems. This guaranteed an increase in the accuracy of the temperature, but also allowed us to recognise erroneous photometric data. As did Pöhnl \& Paunzen (2010), we adopted the temperature calibration by Alonso et al. (1996) for the colour in$\operatorname{dex}(B-V)$, as well as their relation for $(V-R)_{\mathrm{J}}$. Since the colour index is defined for the original Johnson system, and CCD studies are generally performed in the Cousins system, the following transformation by Bessell (1983) needs to be applied:

$(V-R)_{\mathrm{C}}=0.715(V-R)_{\mathrm{J}}-0.02$.

To cover a greater wavelength range, we considered the almost metallicity-independent colour indices $(V-I)$ and $(V-K)$, respectively (see Alonso et al. 1996). This reference provided temperature calibrations for stars cooler than spectral type F0 $(\sim 8000 \mathrm{~K})$. We therefore adopted the colour-temperature relations by Bessell et al. (1998) for $(V-I)_{\mathrm{C}}$ and by Di Benedetto (1998) for $(V-K)_{\mathrm{J}}$, both valid up to about $10000 \mathrm{~K}$. Once again, the latter colour index is in the Johnson system, but since we incorporate 2MASS $K_{\mathrm{s}}$ data for some open clusters, these need the correction given by Carpenter (2001). To extend the $(B-V)$ temperature calibration by Alonso et al. (1996) to stars earlier than spectral type F0, we used the results by Flower (1996). This author provided a compilation of "fundamental" temperatures (for example from Code et al. 1976) for numerous stars of different luminosity classes and derived temperatures based on $(B-V)$ and a calibration for the bolometric correction as a function of colour/temperature. We adopted his list, but used only stars of luminosity class IV/V with available photometric data in other passbands taken from the General Catalogue of Photometric Data (GCPD) $)^{2}$.

Based on a sample of 82 objects that were almost free from reddening, we defined an extension to the temperature calibration by Alonso et al. (1996) for $(V-R)_{\mathrm{C}}$, which is valid for the range between 5000 to $10000 \mathrm{~K}$, with a mean standard deviation $\sigma=140 \mathrm{~K}$ and a correlation coefficient $R=0.993$ (see Fig. 1 and equation below):

$\theta_{\mathrm{eff}}=0.487(3)+0.947(13)(V-R)_{\mathrm{C}}$.

The errors of the last significant digits are given in parentheses. Noteworthy is that the colour index is already in the Cousins system, and $\theta_{\text {eff }}$ is defined as $5040 / T_{\text {eff }}$ in order to avoid a higher order polynomial fit. The fit is in excellent agreement with Alonso et al. (1996) in the overlapping temperature range, with differences of less than $50 \mathrm{~K}$.

For objects hotter than $9500 \mathrm{~K}$ it is more efficient to use reddening-free indices, such as Johnson $Q$ or Strömgren $[u-b]$. We made use of the refined definition $Q=(U-B)-0.71(B-V)$ by Bessell et al. (1998). For completeness, the $[u-b]$ index is defined as $[u-b]=\left[c_{1}\right]+2\left[m_{1}\right]$ with $\left[c_{1}\right]=c_{1}-0.2(b-y)$ and $\left[m_{1}\right]=$ $m_{1}+0.32(b-y)$ according to Crawford \& Mandwewala (1976). As for the temperature relation presented above, we made use of stars listed by Flower (1996), but selected only the hotter ones $(\gtrsim 9500 \mathrm{~K})$. This sample was extended with temperatures for O/B type stars presented in the papers by Morel et al. (2008), Przybilla et al. (2008), Simón-Díaz (2010), and Nieva \& SimónDíaz (2011). Furthermore, we included results by Lefever et al. (2010), however only their "well-studied" objects were adopted (see the reference for details). No significant offsets or trends (within the respective errors) were found between common stars

2 http://obswww. unige.ch/gcpd/gcpd.html 
M. Netopil and E. Paunzen: Towards a photometric metallicity scale for open clusters

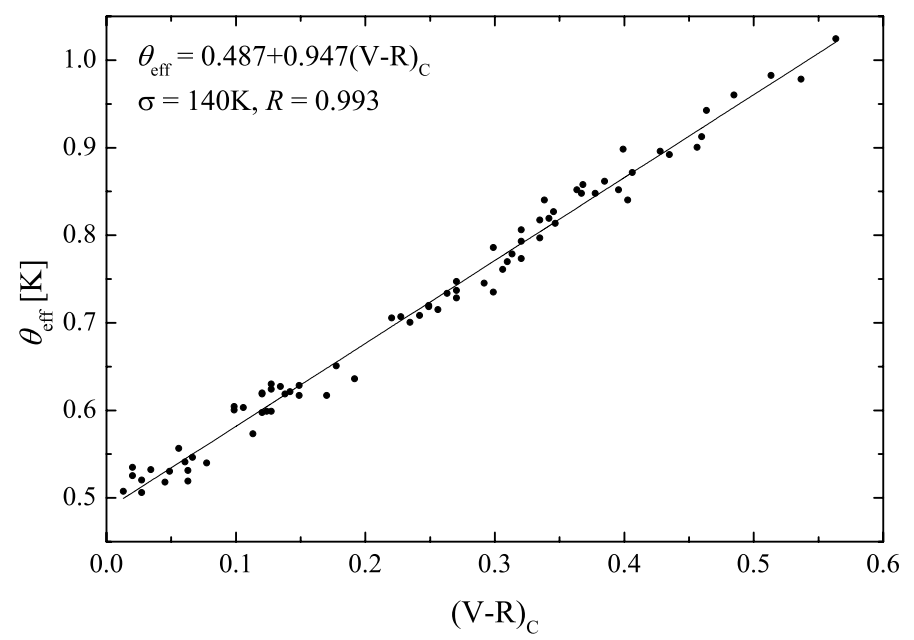

Fig. 1. Empirical temperature calibration for the colour index $(V-R)_{\mathrm{C}}$.

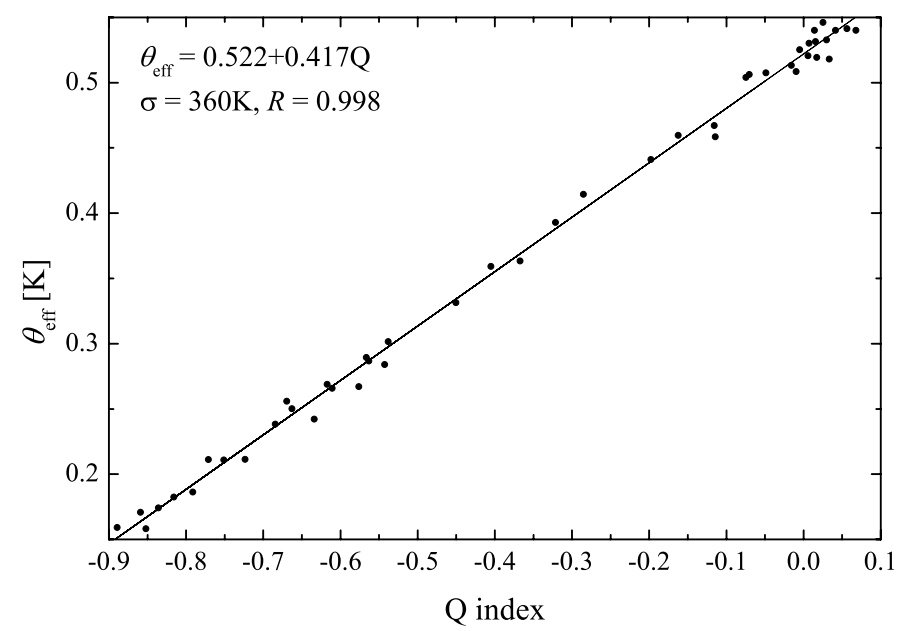

Fig. 2. Empirical temperature calibration for the reddening free $U B V$ $Q$-index.

of the different studies, we therefore adopted mean values for the subsequent analysis. The compiled list of stars was checked for luminosity class (IV/V) and variability, resulting in the exclusion of several $\beta$ Cephei type stars or slowly pulsating B-type objects. In total, 46 objects define our final list of fundamental temperatures. Their relation to the Johnson $Q$ and Strömgren $[u-b]$ indices are presented in Figs. 2 and 3, respectively, with mean standard deviations of about $350 \mathrm{~K}$ and correlation coefficients $R=0.998$ for both empirical calibrations:

$\theta_{\mathrm{eff}}=0.522(2)+0.417(4) Q$

$\theta_{\mathrm{eff}}=0.165(3)+0.286(10)[u-b]-0.021(7)[u-b]^{2}$.

In addition to the temperature calibrations already discussed, we applied the widely accepted routines by Künzli et al. (1997) to the Geneva photometry, and the UVBYBETA code developed by Napiwotzki et al. (1993) to the $u v b y \beta$ data. These authors also provided calibrations in terms of $(b-y)$ and $[u-b]$, the latter based on only 14 objects. A comparison with our $[u-b]$ scale shows that both are in reasonable agreement, although the temperatures by Napiwotzki et al. (1993) around $30000 \mathrm{~K}$ are underestimated by about $2 \%$.

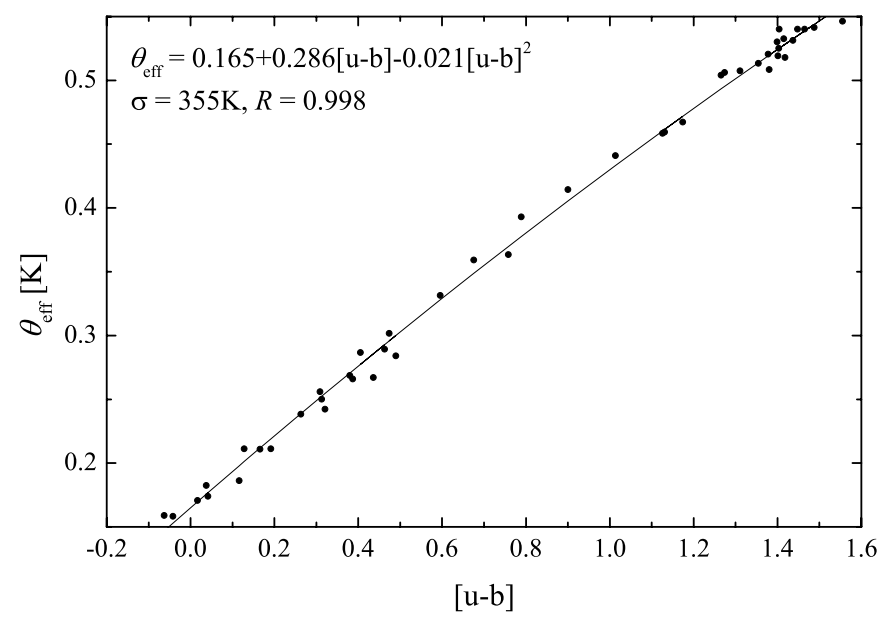

Fig. 3. Empirical temperature calibration for the reddening free $[u-b]$ Strömgren index.

\section{The photometric data and member selection}

The WEBDA ${ }^{3}$ database is probably the most valuable source available for open cluster data (photometry and auxiliary information such as membership probabilities). With the implemented tools, the database also allows for an initial comparison of different datasets. We retrieved almost all available data for our programme clusters from it. Photographic measurements were not directly used for the presented study owing to their high associated uncertainties. However, they were used to verify the other photometry.

To identify possible erroneous datasets, a comparison of all available measurements was conducted. Using the equations by Harmanec \& Božić (2001), we also transformed the photometry between the $U B V, u v b y \beta$, and Geneva systems, in order to check the individual zero points. However, compared to some CCD studies discussed below, the photoelectric studies showed hardly any significant offsets or gradients. Therefore, we used photoelectric data instead of CCD measurements, when the photoelectric data are sufficiently complete down to the lower mass stars.

Paunzen \& Maitzen (2002) noticed that the CCD $V$ measurements for NGC 6451 by Piatti et al. (1998) were too bright compared to other literature results. We determined an offset of 0.97 mag using data by Kjeldsen \& Frandsen (1991). However, the provided $(V-I)_{\mathrm{C}}$ colours by Piatti et al. (1998) were not influenced. This was verified by comparing the resulting I magnitudes to those from the DENIS survey ${ }^{4}$. In a follow-up paper, Paunzen et al. (2003) investigated this cluster and NGC 6192 with uvby filters. The last object has been studied well in $U B V$, so that a comparison using the transformations by Harmanec \& Božić (2001) revealed that the $V$ magnitudes are on the correct scale. However, especially $(u-b)$ was inaccurately standardised, probably owing to the use of only three spectrophotometric standard stars. This led to differing temperatures based on the routine by Napiwotzki et al. (1993) and the $[u-b]$ calibration defined in our study. We therefore excluded the datasets for both clusters.

Kjeldsen \& Frandsen (1991) provided UBV CCD photometry for 13 open clusters in total, with six of them among our programme targets. They mentioned that their transformation

\footnotetext{
3 http://webda.physics . muni.cz

4 VizieR online data catalogue: B/denis.
} 
to standard $(U-B)$ colours is highly inaccurate. We therefore have not incorporated this colour index from the latter reference. The remaining colours appear correctly transformed and were considered for our analysis.

Recently, Glushkova et al. (2013) have investigated the cluster NGC 7296 by means of $B V(R I)_{\mathrm{C}}$ photometry. They notice some offsets in the data by Netopil et al. (2005), by comparing these to their measurements and the ones of the AAVSO Photometric All-Sky Survey (APASS ${ }^{5}$ ). Therefore, instead of correcting the previously available ones, we adopted only the measurements by Glushkova et al. (2013). They offered a much better cluster coverage and additional colour information.

For the open cluster Ruprecht 130, the only available photometric studies are the ones by Piatti et al. (2000) by means of $B V I_{\mathrm{C}}$ and Paunzen et al. (2006). The latter reference used the former to calibrate their $\Delta a$ photometry. Using the Guide Star Catalog for an initial check of the photometry, an offset similar to the one found for NGC 6451 can be seen. According to the observation logs, one can infer that the data presented by Piatti et al. (1998, 2000) were obtained during the same observing run. We therefore applied the same offset to the $V$ magnitudes as determined for NGC 6451 (+0.97 mag). Again, the resulting $I$ magnitudes were compared with the Denis survey, showing excellent agreement ( $\sim 0.01 \mathrm{mag})$. Furthermore, some cluster stars were covered by the APASS survey. The stellar magnitudes corresponded well to the corrected $V$ measurements and the $(B-V)$ colours from Piatti et al. (2000). To obtain the additional colour index $(V-K)$ for the stars in Ruprecht 130 (see Sect. 3), we queried the 2MASS catalogue. However, this cluster is very faint, and data with good ("A" quality flag) photometry are only available for the brightest objects. We therefore used the DR8plus release of the UKIDSS Galactic Plane Survey (GPS, Lucas et al. 2008) for an extension to fainter stars. However, in this area we noticed the following offset and colour dependencies between the UKIDSS and 2MASS photometry. This was based on about 200 common stars with the highest quality flags in both surveys:

$$
\begin{aligned}
J_{2 \mathrm{M}} & =J_{\mathrm{UK}}+0.068(39) \\
(J-H)_{2 \mathrm{M}} & =0.133(6)+1.063(7)(J-H)_{\mathrm{UK}} \\
(J-K)_{2 \mathrm{M}} & =0.092(10)+1.025(8)(J-K)_{\mathrm{UK}} .
\end{aligned}
$$

The UKIDSS GPS survey uses 2MASS in combination with extinction values from the dust maps by Schlegel et al. (1998) to define the photometric zero-points (see Lucas et al. 2008, for details). The reddening value provided by these dust maps is several magnitudes higher than the actual value for the cluster (see Sect. 5). Thus, the UKIDSS photometry is probably influenced in this area. The corrected UKIDSS data were merged with 2MASS photometry to cover the cluster from the brightest to faintest objects. We selected only the most accurate measurements using the photometric quality flags from both datasets.

We note that the aforementioned APASS survey is also still in progress. It currently provides aperture photometry (17" diameter), resulting in crucial blends in denser areas. Taking this limitation into account by selecting isolated stars, we incorporated APASS data for some closer poorly covered clusters such as Trumpler 10 .

Only some photoelectric $U B V$ measurements by Mohan \& Pandey (1984) are available for the brightest stars in the open cluster King 21. We therefore used this dataset, together with

\footnotetext{
5 http://www. aavso.org/apass
}

Table 1. Adopted temperature calibrations.

\begin{tabular}{ll}
\hline \hline Colour/System & Calibration \\
\hline$Q$ index $(U B V)$ & this study \\
$(B-V)$ & Alonso et al. (1996)/Flower (1996) \\
$(V-R)$ & Alonso et al. (1996)/this study ${ }^{a}$ \\
$(V-I)$ & Bessell et al. (1998) \\
$(V-K)$ & Di Benedetto (1998) \\
Geneva & Künzli et al. (1997) \\
uvby $\beta$ & Napiwotzki et al. (1993) \\
$(b-y)$ & Napiwotzki et al. (1993) \\
{$[u-b]$} & this study \\
\hline
\end{tabular}

Notes. ${ }^{(a)}$ For stars cooler/hotter than $\sim 8000 \mathrm{~K}$ as described in the text.

APASS photometry, to recalibrate the $\Delta a$ photometry by Netopil et al. (2007), in order to obtain at least $V$ and $(B-V)$ for the fainter stars. The complete available data set of the APASS survey cannot be safely used for the reason described previously. However, because King 21 is a young cluster and we are not able to derive individual reddening values with the resulting data, a restriction to temperatures lower than $10000 \mathrm{~K}$ was made to avoid erroneous results. Furthermore, we applied calibrated $\Delta a$ photometry for the analysis of NGC 6830, another poorly studied cluster.

Finally, if several studies in the same colour were available for individual cluster stars, their mean values were adopted for the analysis. One exception was the cluster NGC 6705, which was covered by a $B V I_{\mathrm{C}}$ standard field sequence (Stetson 2000). We therefore used only the latter work, combined with $(U-B)$ data from Sung et al. (1999).

As mentioned in Sect. 2, the DG method makes use of main-sequence stars. The most evolved objects (red giants) can be easily recognised in the various colour-magnitude diagrams (CMD), and the remainder of non-main-sequence stars are noticeable during final analysis by their large deviation from the differential grids in luminosity and temperature.

The cluster membership of the individual objects was determined by means of all available CMD and colour-colour diagram combinations. Furthermore, the kinematic membership probabilities and spectral types listed in WEBDA were consulted. In most cases, temperatures were determined using several calibrations. If significant differences are found among individual results, the objects were excluded in order to obtain a sample of only the most probable cluster stars with accurate photometry for further analysis.

Maitzen \& Catalano (1986) proposed that there are two clusters at different distances in the direction of NGC 2451. This has been confirmed by several studies (Hünsch et al. 2004). Since the mean proper motions differ significantly (see e.g. Kharchenko et al. 2005), the respective members can be easily distinguished. We therefore used Tycho- 2 proper motions and additional literature references (e.g. Platais et al. 2001) to extract 30 and 29 members for NGC $2451 \mathrm{~A}$ and B, respectively.

\section{Results and discussion}

Using the temperature calibrations summarised in Table 1, the compiled photometric data for the programme open clusters were transformed into effective temperatures. Whenever possible, individual reddening values for the cluster stars were derived. We used the reddening-ratios listed by Bessell et al. (1998) 
to transform $E(B-V)$ to other colours in the Johnson-Cousins system, and Strömgren and Geneva reddening values were transformed as follows (see e.g. Netopil et al. 2008): $E(B-V)=$ $1.43 E(b-y)=0.84 E[B-V]=1.14 E(B 2-V 1)=0.83 E(B 2-G)$. If the available data did not allow for determining individual reddening values, a mean cluster reddening value was calculated from compiled literature results and used as the starting value. This was also true for the remaining parameters: age and distance.

Finally, averaged temperatures were used to derive the bolometric corrections (Flower 1996) needed to obtain luminosity. In an iterative procedure, the input cluster parameters were altered until the best fit with the grids by Pöhnl \& Paunzen (2010) was found (lowest $\sigma$ over the complete luminosity range). A consistency check of the derived parameter set was performed by fitting Geneva (Lejeune \& Schaerer 2001) and Padova (Marigo et al. 2008) isochrones to the CMDs. It was possible to apply the DG method to 58 clusters in total. For the remaining clusters, there are too few usable photometric data available. However, the majority of the clusters that we did not investigate were simply too young for this method.

The final results are shown in Table 2 and Fig. 7. The DG method provides the overall metallicity $(Z)$, a parameter that is rarely known for open clusters or even single stars. To allow a comparison with other (e.g. spectroscopic) studies, these were transformed into the more common iron abundance ratio $[\mathrm{Fe} / \mathrm{H}]$ as given by Pöhnl \& Paunzen (2010).

We have to note that it is difficult to list a representative error for the fits. Although the standard deviation of $Z$ (as listed in Table 2) can be used as an estimate, also the number of objects, the coverage down to solar luminosity, and the accuracy and number of available photometric datasets have to be considered. A good example is the poorly populated old open cluster NGC 1901. It only has 11 main-sequence member stars that are usable for the metallicity determination. We derived a value of $Z=0.019(2)$; however, the given error is very probably underestimated, because the mean standard deviation of all investigated clusters is twice as large.

From our sample there are ten targets in common with the investigation by Pöhnl \& Paunzen (2010), allowing a direct comparison of the results. There was little deviation between the derived metallicities from the two studies. However, we noticed three clusters whose iron abundances deviate by more than 0.10 dex. For two of them (NGC 2516 and NGC 7092) the discrepancy could be explained by the applied distances. Especially for NGC 2516, the difference between the true distance moduli used amounts to $-0.27 \mathrm{mag}$ (former minus present study). Our distance scale is in line with the comprehensive investigation by An et al. (2007), who used main-sequence fitting with empirically calibrated isochrones. The results for the third strongly deviating cluster (Melotte 20) were probably affected by incorrect effective temperatures derived by Pöhnl \& Paunzen (2010). We noticed that the temperatures of their most luminous objects were underestimated by about $3000 \mathrm{~K}$. This demonstrates the benefit of incorporating several photometric systems, the use of reddening free indices for hotter stars, and individually determined reddening values compared to adopting a single colour index and a uniform reddening value for all objects. The effective temperatures for Melotte 20 stars derived by us, using $U B V$, $u v b y \beta$, and Geneva photometry, are consistent with a mean standard deviation of $150 \mathrm{~K}$. Owing to the lower temperatures for the luminous stars, Pöhnl \& Paunzen (2010) also obtained a much older age for Melotte 20 compared to our analysis and those quoted in the literature so far. No inconsistencies for the other open clusters in common were noticed.

The largest deviation between starting values and final results was found for Ruprecht 130, an open cluster already discussed in Sect. 4. The derived distance of 2000 pc agrees to within $10 \%$ of the previous values determined by Piatti et al. (2000) and Paunzen et al. (2006), although these values are based on $\sim 1$ mag brighter $V$ magnitudes and a 0.20 mag higher reddening value. However, the most outstanding difference was noticed for the age. Our analysis resulted in a cluster age of $560 \mathrm{Myr}$, whereas the previous studies obtained ages of 50 and 80 Myr. Both studies performed isochrone fitting, however Piatti et al. (2000) also matched integrated spectra to available templates. Figure 4 shows the NIR and magnitude corrected visual CMD overlayed with solar composition isochrones by Marigo et al. (2008). The apparent distance modulus given by Piatti et al. (2000) was corrected to account for the derived magnitude offset. For the NIR isochrones, the parameters were transformed using the relations $E\left(J-K_{\mathrm{S}}\right)=0.488 E(B-V)$ by Bonatto et al. (2004) and $A_{K_{\mathrm{S}}}=0.67 E\left(J-K_{\mathrm{S}}\right)$ by Dutra et al. (2002).

It is obvious that in the visual region the previous parameters do not provide a proper fit for the main-sequence at all. Furthermore, the age is set by the brightest and bluest objects. In contrast, our determined parameters cover the probable red giant branch, noticeable especially in the NIR diagram. We would like to draw attention to the fact that the results of the DG method are based solely on the main-sequence. In light of the older age, the aforementioned brightest and bluest objects can be assigned to the blue hook or to the group of blue stragglers.

\subsection{The results in light of lower solar abundances}

The isochrones by Pöhnl \& Paunzen (2010) are standardised on evolutionary grids with the previously accepted solar metallicity $Z=0.020$ (Anders \& Grevesse 1989). However, the solar value was meanwhile scaled down to $Z=0.0134$ by Asplund et al. (2009). Using a reduced solar metal content, Mowlavi et al. (2012) present new stellar models ranging from $Z=0.006$ to 0.040 . Unfortunately, these were provided after the beginning of our investigation, and the models are currently only available up to $3.5 M_{\odot}$. Nevertheless, it is worth investigating the influence of the different metallicity scales. Therefore, we searched for a suitable open cluster with sufficient photometry and well known parameters. The aforementioned mass restriction of the new models limits one to clusters with an age of $\log t \sim 8.3$, in order to be able to cover the complete main-sequence and the widest possible range in luminosity. Among our programme clusters, NGC 6475 was a good candidate for this comparison.

Following Pöhnl \& Paunzen (2010), we constructed new differential grids based on the ones by Mowlavi et al. (2012) with their $Z=0.014$ model as a reference. This was performed for ages around our final result of $\log t=8.4$, which is in very good agreement with the starting value $(\log t=8.34)$ compiled from the literature. The best fit (Fig. 5) was obtained with the same age $(\log t=8.4)$ and $Z=0.017(4)$. Using Eq. (A.5) by Mowlavi et al. (2012) this resulted in a metallicity of $[\mathrm{Fe} / \mathrm{H}]=0.14 \mathrm{dex}$, whereas the original grids gave a slightly lower value of $[\mathrm{Fe} / \mathrm{H}]=0.11$ dex derived using $Z=0.024(5)$. The applied distances agree to within a few percent; $263 \mathrm{pc}$ (new grid) vs. $269 \mathrm{pc}$ (old grid). Both of them are very close to the Hipparcos based result of 270 pc (van Leeuwen 2009). One can conclude that the DG method is independent of the grids used. The resulting $Z$ values only need to be transformed into the corresponding $[\mathrm{Fe} / \mathrm{H}]$ values. Our results are in good agreement 
Table 2. Results for the programme targets.

\begin{tabular}{|c|c|c|c|c|c|c|c|}
\hline Cluster & $\log t$ & $(m-M)_{0}$ & $E(B-V)^{a}$ & $Z$ & {$[\mathrm{Fe} / \mathrm{H}]_{\mathrm{DG}}$} & {$[\mathrm{Fe} / \mathrm{H}]_{\mathrm{spec}^{b}}{ }^{b}$} & Phot. systems ${ }^{c}$ \\
\hline Berkeley 11 & 7.80 & 12.20 & $0.96(6)$ & $0.020(5)$ & $+0.01(14)$ & & $U B V_{\mathrm{pe}}, U B V R I_{\mathrm{ccd}}$ \\
\hline Collinder 140 & 7.55 & 8.05 & $0.04(4)$ & $0.021(3)$ & $+0.04(7)$ & & $U B V_{\mathrm{pe}}, \mathrm{Str}_{\mathrm{pe}}, \mathrm{G}$ \\
\hline Collinder 272 & 7.30 & 11.75 & $0.43(3)$ & $0.021(4)$ & $+0.06(9)$ & & $U B V R I_{\mathrm{ccd}}$ \\
\hline IC 2391 & 7.65 & 5.80 & $0.01(1)$ & $0.018(3)$ & $-0.03(7)$ & $-0.02(1) / 2$ & $U B V_{\mathrm{pe}}, \mathrm{Str}_{\mathrm{pe}}, \mathrm{G}$ \\
\hline IC 2602 & 7.85 & 5.85 & $0.03(2)$ & $0.019(3)$ & $+0.00(8)$ & $-0.03(4) / 2$ & $U B V_{\mathrm{pe}}, \mathrm{Str}_{\mathrm{pe}}, \mathrm{G}$ \\
\hline IC 4665 & 7.70 & 7.70 & $0.19(3)$ & $0.020(2)$ & $+0.02(6)$ & $-0.03(4) / 1$ & $U B V_{\mathrm{pe}}, \mathrm{Str}_{\mathrm{pe}}, \mathrm{G}$ \\
\hline IC 4725 & 7.85 & 9.00 & $0.45(5)$ & $0.020(3)$ & $+0.03(8)$ & $+0.02(2) / 1$ & $U B V_{\mathrm{pe}}, \mathrm{Str}_{\mathrm{pe}}$ \\
\hline King 21 & 7.30 & 12.40 & $0.86(6)$ & $0.021(6)$ & $+0.06(14)$ & & $U B V_{\mathrm{pe}}, \Delta a, 2 \mathrm{M}$ \\
\hline Lynga 1 & 8.10 & 11.40 & $0.45(4)$ & $0.021(4)$ & $+0.04(10)$ & & $U B V_{\mathrm{pe}}, U B V R I_{\mathrm{ccd}}$ \\
\hline Melotte 20 & 7.75 & 6.15 & $0.09(3)$ & $0.021(5)$ & $+0.05(13)$ & $+0.09(20) / 2$ & $U B V_{\mathrm{pe}}, \mathrm{Str}_{\mathrm{pe}}, \mathrm{G}$ \\
\hline Melotte 22 & 8.05 & 5.50 & $0.04(2)$ & $0.016(2)$ & $-0.08(7)$ & $+0.02(4) / 5$ & $U B V_{\mathrm{pe}}, \mathrm{Str}_{\mathrm{pe}}, \mathrm{G}$ \\
\hline Melotte 105 & 8.60 & 11.85 & $0.44(4)$ & $0.022(5)$ & $+0.08(12)$ & & $U B V I_{\mathrm{ccd}}, \operatorname{Str}_{\mathrm{ccd}}$ \\
\hline Melotte 111 & 8.85 & 4.70 & $0.00(1)$ & $0.018(2)$ & $-0.04(5)$ & $+0.01(8) / 2$ & $U B V_{\mathrm{pe}}, \mathrm{Str}_{\mathrm{pe}}, \mathrm{G}$ \\
\hline NGC 1039 & 8.25 & 8.35 & $0.08(2)$ & $0.021(4)$ & $+0.05(10)$ & $+0.07(4) / 1$ & $U B V_{\mathrm{pe}}, \mathrm{BV}_{\mathrm{ccd}}, \mathrm{Str}_{\mathrm{pe}}, \mathrm{G}$ \\
\hline NGC 1662 & 8.60 & 8.05 & $0.32(3)$ & $0.021(3)$ & $+0.05(8)$ & & $U B V_{\mathrm{pe}}, \mathrm{Str}_{\mathrm{pe}}, \mathrm{G}$ \\
\hline NGC 1901 & 8.90 & 8.20 & $0.03(2)$ & $0.019(2)$ & $+0.01(5)$ & $-0.08(1) / 1$ & $U B V_{\mathrm{pe}}, U B V_{\mathrm{ccd}}, \mathrm{Str}_{\mathrm{pe}}$ \\
\hline NGC 2099 & 8.65 & 10.55 & $0.30(1)$ & $0.019(2)$ & $+0.00(4)$ & $+0.01(5) / 1$ & $U B V_{\mathrm{pe}}$ \\
\hline NGC 2232 & 7.70 & 7.65 & $0.03(2)$ & $0.025(6)$ & $+0.14(12)$ & $+0.22(9) / 1$ & $U B V_{\mathrm{pe}}, \mathrm{Str}_{\mathrm{pe}}, \mathrm{G}$ \\
\hline NGC 2287 & 8.40 & 9.10 & $0.02(2)$ & $0.014(3)$ & $-0.16(9)$ & $-0.23(2) / 1$ & $U B V_{\mathrm{pe}}, \mathrm{Str}_{\mathrm{pe}}, \mathrm{G}$ \\
\hline NGC 2343 & 8.05 & 9.90 & $0.20(2)$ & $0.021(6)$ & $+0.05(14)$ & & $U B V_{\mathrm{pe}}, \mathrm{by}_{\mathrm{ccd}}$ \\
\hline NGC 2422 & 8.15 & 8.50 & $0.09(2)$ & $0.023(4)$ & $+0.09(8)$ & & $U B V_{\mathrm{pe}}, \mathrm{UBVI}_{\mathrm{ccd}}, \mathrm{Str}_{\mathrm{pe}}, \mathrm{G}$ \\
\hline NGC 2423 & 9.00 & 9.80 & $0.10(1)$ & $0.024(5)$ & $+0.11(10)$ & $+0.14(6) / 1$ & $\mathrm{Str}_{\mathrm{pe}}, \mathrm{G}, \mathrm{APASS}$ \\
\hline NGC 2447 & 8.75 & 10.13 & $0.01(1)$ & $0.020(3)$ & $+0.03(7)$ & $-0.03(6) / 4$ & $\operatorname{Str}_{\mathrm{pe}}, \mathrm{G}$ \\
\hline NGC 2451A & 7.70 & 6.35 & $0.01(1)$ & $0.017(4)$ & $-0.06(11)$ & & $U B V_{\mathrm{pe}}, \mathrm{BV}_{\mathrm{ccd}}, \mathrm{Str}_{\mathrm{pe}}, \mathrm{G}$ \\
\hline NGC 2451B & 7.70 & 7.95 & $0.10(3)$ & $0.020(3)$ & $+0.02(9)$ & & $U B V_{\mathrm{pe}}, \mathrm{Str}_{\mathrm{pe}}, \mathrm{G}$ \\
\hline NGC 2489 & 8.75 & 11.30 & 0.31 & $0.022(4)$ & $+0.06(10)$ & & $U B V I_{\mathrm{ccd}}$ \\
\hline NGC 2516 & 8.25 & 8.05 & $0.11(3)$ & $0.020(5)$ & $+0.03(12)$ & $+0.01(7) / 1$ & $U B V_{\mathrm{pe}}, \mathrm{Str}_{\mathrm{pe}}, \mathrm{G}$ \\
\hline NGC 2546 & 8.15 & 9.75 & $0.14(3)$ & $0.020(4)$ & $+0.01(9)$ & & $U B V_{\mathrm{pe}}$, APASS, $2 \mathrm{M}$ \\
\hline NGC 2567 & 8.45 & 11.05 & $0.13(3)$ & $0.020(4)$ & $+0.02(10)$ & $+0.00(5) / 1$ & $U B V_{\mathrm{pe}}$ \\
\hline NGC 2632 & 8.80 & 6.30 & $0.01(1)$ & $0.027(5)$ & $+0.17(9)$ & $+0.14(10) / 4$ & $U B V_{\mathrm{pe}}, \mathrm{Str}_{\mathrm{pe}}, \mathrm{G}$ \\
\hline NGC 2658 & 8.50 & 13.05 & $0.36(3)$ & $0.020(5)$ & $+0.02(12)$ & & $U B V R I_{\mathrm{ccd}}$ \\
\hline NGC 3114 & 8.20 & 9.85 & $0.07(2)$ & $0.022(5)$ & $+0.07(11)$ & $+0.04(2) / 2$ & $U B V_{\mathrm{pe}}, \mathrm{Str}_{\mathrm{pe}}, \mathrm{G}$ \\
\hline NGC 3228 & 7.85 & 8.35 & $0.04(1)$ & $0.020(4)$ & $+0.03(10)$ & & $U B V_{\mathrm{pe}}$, Genf \\
\hline NGC 3532 & 8.55 & 8.35 & $0.04(2)$ & $0.021(3)$ & $+0.04(7)$ & $+0.05(4) / 3$ & $U B V_{\mathrm{pe}}, \mathrm{Str}_{\mathrm{pe}}, \mathrm{G}$ \\
\hline NGC 3960 & 9.00 & 11.65 & 0.29 & $0.020(4)$ & $+0.03(9)$ & $+0.02(4) / 1$ & $\mathrm{BV}_{\mathrm{pe}}, U B V I_{\mathrm{ccd}}$ \\
\hline NGC 5281 & 7.90 & 10.70 & $0.22(1)$ & $0.019(4)$ & $+0.00(11)$ & & $U B V_{\mathrm{pe}}, B V I_{\mathrm{ccd}}$ \\
\hline NGC 5460 & 8.20 & 9.20 & $0.13(1)$ & $0.021(4)$ & $+0.06(9)$ & $+0.05(24) / 1$ & $U B V_{\mathrm{pe}}, \mathrm{Str}_{\mathrm{pe}}, \mathrm{G}$ \\
\hline NGC 5662 & 8.05 & 9.30 & $0.30(4)$ & $0.022(4)$ & $+0.06(9)$ & & $U B V_{\mathrm{pe}}, \mathrm{Str}_{\mathrm{pe}}$ \\
\hline NGC 5999 & 8.70 & 11.75 & 0.46 & $0.019(3)$ & $+0.00(8)$ & & $B V_{\mathrm{pe}}, B V I_{\mathrm{ccd}}$ \\
\hline NGC 6031 & 8.40 & 11.00 & $0.44(3)$ & $0.020(4)$ & $+0.02(9)$ & & $U B V_{\mathrm{pe}}, B V I_{\mathrm{ccd}}$ \\
\hline NGC 6087 & 7.95 & 9.60 & $0.19(2)$ & $0.022(5)$ & $+0.06(12)$ & $+0.06(20) / 1$ & $U B V_{\mathrm{pe}}, \mathrm{Str}_{\mathrm{pe}}$ \\
\hline NGC 6134 & 9.00 & 10.00 & $0.40(3)$ & $0.026(5)$ & $+0.16(9)$ & $+0.14(2) / 3$ & $U B V_{\mathrm{pe}}, B V_{\mathrm{ccd}}, \mathrm{Str}_{\mathrm{ccd}}$ \\
\hline NGC 6192 & 8.20 & 11.05 & $0.63(4)$ & $0.026(7)$ & $+0.16(13)$ & $+0.12(4) / 1$ & $U B V_{\mathrm{pe}}, B V_{\mathrm{ccd}}, 2 \mathrm{M}$ \\
\hline NGC 6204 & 8.05 & 10.25 & $0.47(1)$ & $0.021(5)$ & $+0.05(12)$ & & $U B V_{\mathrm{pe}}, B V I_{\mathrm{ccd}}, \mathrm{by}_{\mathrm{ccd}}$ \\
\hline NGC 6281 & 8.40 & 8.65 & $0.17(3)$ & $0.021(3)$ & $+0.04(7)$ & $+0.05(6) / 1$ & $U B V_{\mathrm{pe}}, \mathrm{Str}_{\mathrm{pe}}, \mathrm{G}$ \\
\hline NGC 6405 & 7.90 & 8.45 & $0.17(2)$ & $0.023(5)$ & $+0.09(11)$ & & $U B V_{\mathrm{pe}}, \mathrm{Str}_{\mathrm{pe}}, \mathrm{G}$ \\
\hline NGC 6451 & 8.00 & 11.75 & 0.75 & $0.021(4)$ & $+0.04(10)$ & & $B V I_{\mathrm{ccd}}$ \\
\hline NGC 6475 & 8.40 & 7.15 & $0.07(3)$ & $0.024(5)$ & $+0.11(11)$ & $+0.09(8) / 2$ & $U B V_{\mathrm{pe}}, \mathrm{Str}_{\mathrm{pe}}, \mathrm{G}$ \\
\hline NGC 6705 & 8.40 & 11.60 & $0.39(3)$ & $0.027(5)$ & $+0.18(8)$ & $+0.17(9) / 2$ & $U B V I_{\mathrm{ccd}}$ \\
\hline NGC 6756 & 8.10 & 12.30 & $1.03(5)$ & $0.023(6)$ & $+0.10(14)$ & & $\operatorname{Str}_{\mathrm{ccd}}$ \\
\hline NGC 6802 & 9.00 & 11.65 & 0.79 & $0.020(5)$ & $+0.03(13)$ & & $B V I_{\mathrm{ccd}}$ \\
\hline NGC 6830 & 8.10 & 11.90 & $0.54(4)$ & $0.031(3)$ & $+0.24(5)$ & & $U B V_{\mathrm{pe}}, \Delta a, 2 \mathrm{M}$ \\
\hline NGC 7092 & 8.55 & 7.45 & $0.03(2)$ & $0.020(3)$ & $+0.02(9)$ & & $U B V_{\mathrm{pe}}, \mathrm{Str}_{\mathrm{pe}}, \mathrm{G}$ \\
\hline NGC 7243 & 8.00 & 9.45 & $0.24(3)$ & $0.021(6)$ & $+0.06(13)$ & & $U B V_{\mathrm{pe}}, \mathrm{Str}_{\mathrm{pe}}, \mathrm{G}$ \\
\hline NGC 7296 & 8.55 & 12.15 & 0.20 & $0.019(5)$ & $+0.00(13)$ & & $B V R I_{\mathrm{ccd}}$ \\
\hline Ruprecht 115 & 8.65 & 11.35 & 0.74 & $0.021(3)$ & $+0.04(7)$ & & $B V I_{\mathrm{ccd}}$ \\
\hline Ruprecht 130 & 8.75 & 11.50 & 1.00 & $0.020(5)$ & $+0.03(14)$ & & $B V I_{\mathrm{ccd}}, 2 \mathrm{M} / \mathrm{UKIDSS}$ \\
\hline Trumpler 10 & 7.80 & 8.05 & $0.03(1)$ & $0.016(3)$ & $-0.07(10)$ & & $U B V_{\mathrm{pe}}$, APASS, $\mathrm{Str}_{\mathrm{pe}}, \mathrm{G}$ \\
\hline
\end{tabular}

Notes. ${ }^{(a)}$ The standard deviation of reddening is given in parentheses, if reddening was determined via photometric calibrations (e.g. $U B V-$ $Q$ method). ${ }^{(b)}$ The mean spectroscopic $[\mathrm{Fe} / \mathrm{H}]$ ratio and the standard deviation in parentheses. The number of studies used (listed by Carrera $\&$ Pancino 2011, and additional literature) is given by /\#. ${ }^{(c)}$ Photometric systems or surveys used for the analysis, e.g. G (Geneva), Str (Strömgren), 2M (2MASS), whereas pe/ccd stands for photoelectric and CCD data, respectively. For some clusters, $V /(b-y)$ data by McSwain \& Gies $(2008)$ were included (by $\mathrm{ccd})$, or $\Delta a$ photometry transformed to $V /(B-V)$ as discussed in Sect. 4. 
M. Netopil and E. Paunzen: Towards a photometric metallicity scale for open clusters
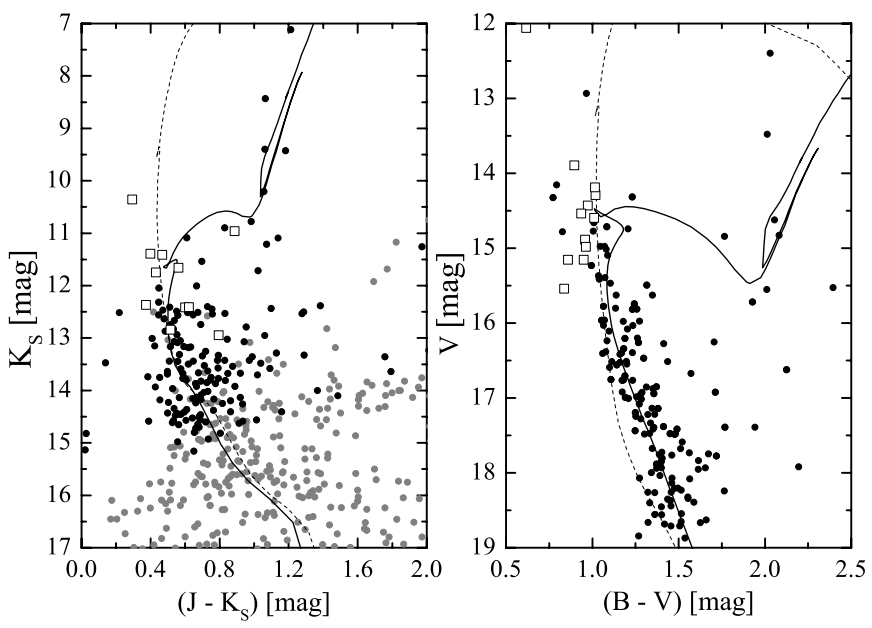

Fig. 4. Colour-magnitude diagrams for Ruprecht 130. Black dots in the NIR CMD represent the stars observed in the visual by Piatti et al. (2000). Grey dots are additional 2MASS/UKIDSS objects within a $2^{\prime}$ radius. Open squares indicate the position of stars near the blue hook and probable blue stragglers, which are noticeable in the visual, with available good quality NIR data. The solid lines show the isochrones for our results (560 Myr), and the dashed lines $50 \mathrm{Myr}$ isochrones with the parameters listed by Piatti et al. (2000).

with the mean spectroscopic iron abundance of 0.09(8) dex, which was derived from two sources: $[\mathrm{Fe} / \mathrm{H}]=0.14(6)$ dex by Sestito et al. (2003) and 0.03(2) dex by Villanova et al. (2009). Differing metallicities are also found for other open clusters (e.g. Melotte 20 or NGC 2632). By using the spectral resolution and the number of investigated stars as criteria to determine the most reliable result, the higher value by Sestito et al. (2003) should be considered.

\section{Comparison with spectroscopic results}

Carrera \& Pancino (2011) present new abundance determinations for a number of open clusters, as well as a comprehensive list of high-resolution spectroscopic $[\mathrm{Fe} / \mathrm{H}]$ results compiled from the literature. We used this list as a reference, along with studies by An et al. (2007), Pancino et al. (2010), Schuler et al. (2010), and Santos et al. (2012). All measurements were based on spectroscopic data with a resolution of at least $R=16000$, however the majority were obtained with $R=40000$ or higher. Where several studies were available for one cluster, we calculated a mean $[\mathrm{Fe} / \mathrm{H}]$ value and its standard deviation. Otherwise, we adopted the individual results and quoted errors from these references.

For 27 programme open clusters, spectroscopic determinations were found in the literature. Furthermore, we included the DG results for three open clusters (Melotte 25, NGC 752, and Berkeley 29) investigated by Pöhnl \& Paunzen (2010), due to the availability of spectroscopic data for them. The last cluster is of particular interest, because it is the most underabundant aggregate in the complete sample so far. This allowed for a better verification of the metallicity scale obtained by the DG method (see Fig. 6).

The largest deviation ( 0.15 dex) between spectroscopic results and our determination was initially found for IC 4725 . The iron abundance $[\mathrm{Fe} / \mathrm{H}]=0.18(8)$ dex was derived by Luck (1994), as listed by Carrera \& Pancino (2011). However, for the three investigated cluster stars, Luck et al. (2000) present revised metallicities based on spectra with much higher

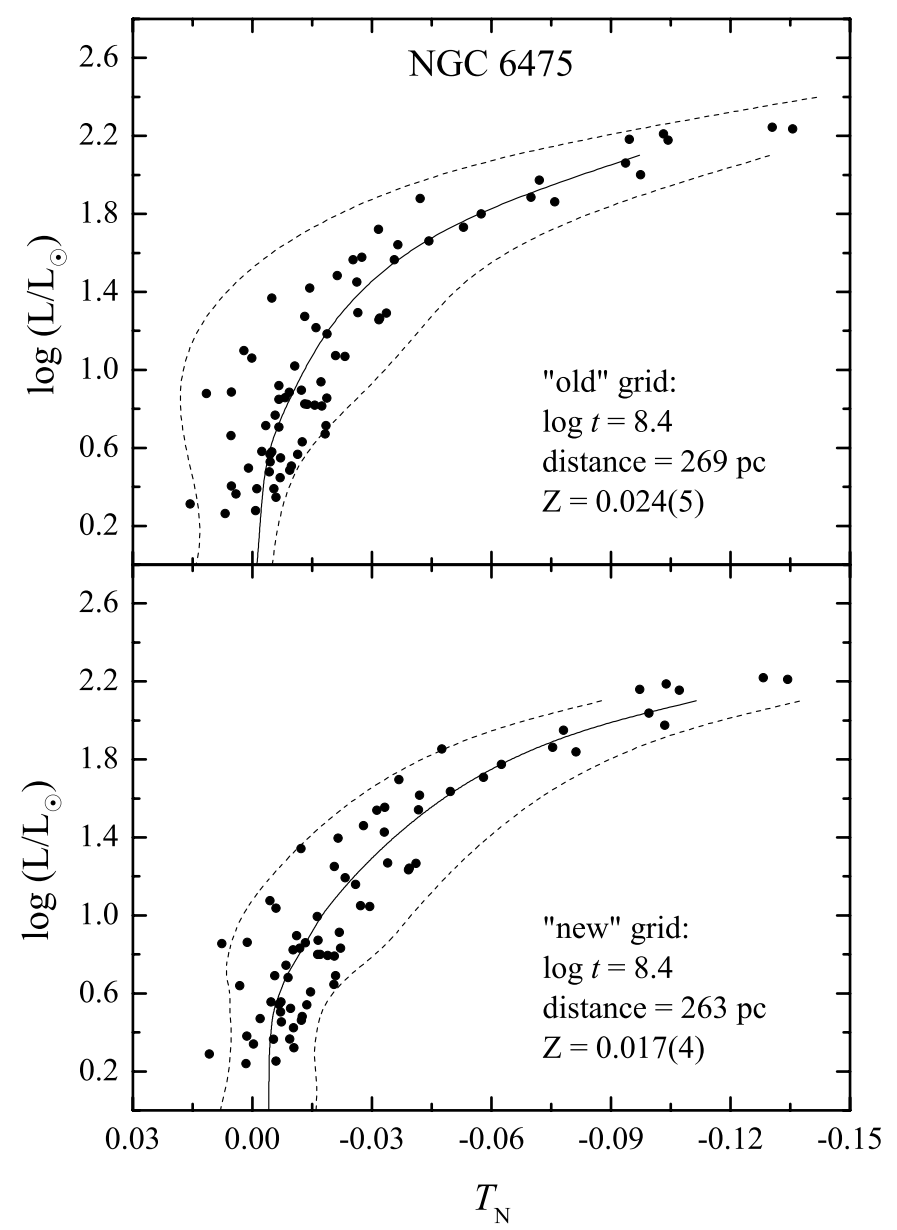

Fig. 5. Comparison of the old and new differential grids applied to NGC 6475. Additionally, some isochrones for the same age but for different metallicities are shown for a comparison with the dashed lines: $Z=0.010 / 0.040$ (old grid) and $Z=0.010 / 0.030$ (new grid). The effective temperature is normalised $\left(T_{\mathrm{N}}\right)$ to the ZAMS of the respective solar metallicity.

resolution $(R=60000$ compared to $R=18000)$. The resulting mean $[\mathrm{Fe} / \mathrm{H}]=0.02(2)$ dex is in excellent agreement with our study $([\mathrm{Fe} / \mathrm{H}]=0.03(8)$ dex $)$. Somewhat less deviating results were found for the open clusters Melotte 22 (Pleiades) and NGC 1901 with differences of 0.10 and 0.09 dex, respectively. The spectroscopic determination for NGC 1901 was based on a single star analysed by Carraro et al. (2007). The discrepancy for the prominent and well investigated Pleiades cluster could be due to the transformation of the overall metallicity $Z$ to $[\mathrm{Fe} / \mathrm{H}]$, assuming a correlation between the various abundance ratios. The analysis by Gebran \& Monier (2008) showed a deficiency in the main contributors to $Z$ : the elements $\mathrm{C}$ and $\mathrm{O}$. Therefore, our derived iron abundance of $[\mathrm{Fe} / \mathrm{H}]=-0.08(7)$ dex for the Pleiades is also very probably underestimated.

Using the complete sample of 30 open clusters with available spectroscopic metallicity determinations, and adopting the revised result for IC 4725, we concluded that the DG method matches the spectroscopic scale very well (Fig. 6). The mean value of the absolute deviations amounts to $0.03(3)$ dex, without a noticeable offset or gradient. However, more aggregates in the underabundant regime are still necessary for a more detailed comparison. 


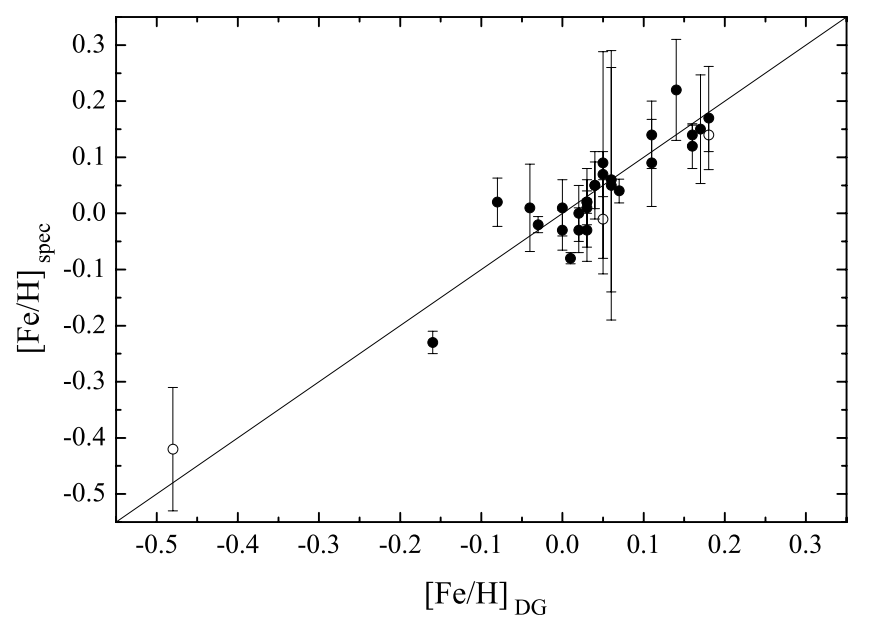

Fig. 6. Comparison of our metallicity determinations to high-resolution spectroscopic values from the literature. Open symbols are additional open clusters by Pöhnl \& Paunzen (2010), and the straight line represents the one-to-one relation. The errors of our results are about 0.1 dex for most clusters, but were omitted for better visibility.

\section{Conclusion}

We examined 58 open clusters in total using the differential grid method introduced by Pöhnl \& Paunzen (2010). The comparison of the derived cluster metallicities with spectroscopic metallicity determinations showed that accurate results could be achieved. Although the mean deviations to spectroscopic studies were lower than $0.05 \mathrm{dex}$ for $[\mathrm{Fe} / \mathrm{H}]$, the comparison with results by Pöhnl \& Paunzen (2010) showed that improperly applied distances can lead to errors that are somewhat larger ( $\sim 0.1$ dex). However, differences of that order are also not uncommon among spectroscopic studies. Examining the compilation by Carrera \& Pancino (2011), we found results with much greater discrepancies (e.g. for Melotte 20, NGC 2632, or Collinder 261).

To verify the effect of the currently accepted solar abundance value (Asplund et al. 2009), the result for NGC 6475 using the original differential grids by Pöhnl \& Paunzen (2010) were compared with newly constructed grids based on the recent evolutionary models by Mowlavi et al. (2012). Since the derived parameters were in excellent agreement, we concluded that the influence of a different solar metallicity $(Z=0.02$ compared to $Z=0.014)$ is negligible. The resulting metallicity values only need to be properly transformed into $[\mathrm{Fe} / \mathrm{H}]$ values. However, more comparisons will be needed as soon as the new models are extended to higher masses.

Nevertheless, this method provides a robust way of obtaining accurate metallicity estimates for large samples of open clusters, necessary for a more in-depth study of various relationships, such as metallicity gradients or age dependencies. The future availability of deep and homogeneous photometric surveys (e.g. LSST, Pan-STARRS, Vista) will allow the study of cluster metallicities in the very inner and outer regions of the Milky Way in a consistent way. Spectroscopic investigations, on the other hand, are very time-consuming, therefore only about 100 open clusters are covered so far. Furthermore, for most targets, the overall cluster metallicity has been defined by three or less stars (as a rule red giants, the brightest objects). The influence of improper membership determination in these number limited samples must also be considered.

As soon as accurate astrophysical parameters are known for numerous individual cluster stars, the DG method allows evolutionary models to be tested. In this respect we would like to mention the upcoming Gaia satellite mission, which will provide spectroscopic effective temperatures and metallicities (limited in distance and temperature domain), as well as parallax, radial velocity, and proper motion data, to identify true open cluster members and to derive luminosity. With these data sets at hand, our method can be extended to investigate topics related with rotation. This parameter was recently implemented in the models by the Geneva group (Ekström et al. 2012). Furthermore, an extension of the differential grids to post main-sequence stages will be helpful when investigating older stellar aggregates (e.g. globular clusters) in more detail.

The investigated target sample comes from the $\Delta a$ photometric survey (e.g. Netopil et al. 2007), which aims to detect chemically peculiar stars in open clusters. In a follow-up study, the results of the present paper will be used to investigate the dependencies of this star group with age and metallicity.

Acknowledgements. This publication makes use of data products from the Two Micron All Sky Survey, which is a joint project of the University of Massachusetts and the Infrared Processing and Analysis Center/California Institute of Technology, funded by the National Aeronautics and Space Administration and the National Science Foundation, and was made possible through the use of the AAVSO Photometric All-Sky Survey (APASS), funded by the Robert Martin Ayers Sciences Fund. Furthermore, this research has made use of the WEBDA database, operated at the Department of Theoretical Physics and Astrophysics of the Masaryk University. This work was supported by the grants GA ČR P209/12/0217, 7AMB12AT003, the Austrian Research Fund via the project FWF P22691-N16, and the financial contributions of the Austrian Agency for International Cooperation in Education and Research (CZ-10/2012). We would like to thank Kieran Leschinski for his help with language editing.

\section{References}

Alonso, A., Arribas, S., \& Martínez-Roger, C. 1996, A\&A, 313, 873 An, D., Terndrup, D. M., Pinsonneault, M. H., et al. 2007, ApJ, 655, 233 Anders, E., \& Grevesse, N. 1989, Geochim. Cosmochim. Acta, 53, 197 Asplund, M., Grevesse, N., Sauval, A. J., \& Scott, P. 2009, ARA\&A, 47, 481 Bessell, M. S. 1983, PASP, 95, 480

Bessell, M. S., Castelli, F., \& Plez, B. 1998, A\&A, 333, 231

Bonatto, C. H., Bica, E., \& Girardi, L. 2004, A\&A, 415, 571

Carpenter, J. M. 2001, AJ, 121, 2851

Carraro, G., de La Fuenta, M. R., Villanova, S., et al. 2007, A\&A, 466, 931

Carrera, R., \& Pancino, E. 2011, A\&A, 535, A30

Code, A. D., Davis, J., Bless, R. C., \& Hanbury Brown, R. 1976, ApJ, 203, 417 Cramer, N. 1982, A\&A, 112, 330

Crawford, C. L., \& Mandwewala N. 1976, PASP, 88, 917

Dias, W. S., Alessi, B. S., Moitinho, A., \& Lépine, J. R. D. 2002, A\&A, 389, 871 Di Benedetto, G. P. 1998, A\&A, 339, 858

Dutra, C. M., Santiago, B. X., \& Bica, E. 2002, A\&A, 381, 219

Ekström, S., Georgy, C., Eggenberger, P., et al. 2012, A\&A, 537, A146

Flower, P. J. 1996 ApJ, 469, 355

Gebran, M., \& Monier, R. 2008, A\&A, 483, 567

Glushkova, E. V., Zabolotskikh, M. V., Koposov, S. E., et al. 2013, MNRAS, 429, 1102

Harmanec, P., \& Božić, H. 2001, A\&A, 369, 1140

Hünsch, M., Randich, S., Hempel, M., Weidner, C., \& Schmitt, J. H. M. M. 2004 A\&A, 418, 539

Johnson, H. L. 1958, Lowell Observatory, 4, 37

Kharchenko, N. V., Piskunov, A. E., Röser, S., Schilbach, E., \& Scholz, R.-D. 2005, A\&A, 438, 1163

Kjeldsen, H., \& Frandsen, S. 1991, A\&AS, 87, 119

Künzli, M., North, P., Kurucz, R. L., \& Nicolet, B. 1997, A\&AS, 122, 51

Lefever, K., Puls, J., Morel, T., et al. 2010, A\&A, 515, A74

Lejeune, T., \& Schaerer, D. 2001, A\&A, 366, 538

Lucas, P. W., Hoare, M. G., Longmore, A., et al. 2008, MNRAS, 391, 136

Luck, R. E. 1994, ApJS, 91, 309

Luck, R. E., Andrievsky, S. M., Kovtyukh, V. V., Korotin, S. A., \& Beletsky, Yu. V. 2000, A\&A, 361, 189

Maitzen, H. M., \& Catalano, F. A. 1986, A\&AS, 66, 37

Marigo, P., Girardi, L., Bressan, A., et al. 2008, A\&A, 482, 883

McSwain, M. V., \& Gies, D. R. 2005, ApJS, 161, 118

Mohan, V., \& Pandey, A. K. 1984, BASI, 12, 217 
M. Netopil and E. Paunzen: Towards a photometric metallicity scale for open clusters

Morel, T., Hubrig, S., \& Briquet, M. 2008, A\&A, 481, 453

Mowlavi, N., Eggenberger, P., Meynet, G., et al. 2012, A\&A, 541, A41

Napiwotzki, R., Schoenberner, D., \& Wenske, V. 1993, A\&A, 268, 653

Netopil, M., Paunzen, E., Maitzen, H. M., et al. 2005, Astron. Nachr., 326, 734

Netopil, M., Paunzen, E., Maitzen, H. M., et al. 2007, A\&A, 462, 591

Netopil, M., Paunzen, E., Maitzen, H. M., North, P., \& Hubrig, S. 2008, A\&A, 491, 545

Nieva, M.-F., \& Simón-Díaz, S. 2011, A\&A, 532, A2

Pancino, E., Carrera, R., Rossetti, E., \& Gallart, C. 2010, A\&A, 511, A56

Paunzen, E., \& Maitzen, H. M. 2002, A\&A, 385, 867

Paunzen, E., \& Netopil, M. 2006, MNRAS, 371, 1641

Paunzen, E., Maitzen, H. M., Rakos, K. D., \& Schombert, J. 2003, A\&A, 403, 937

Paunzen, E., Netopil, M., Iliev, I. Kh., et al. 2006, A\&A, 454, 171

Paunzen, E., Heiter, U., Netopil M., \& Soubiran C. 2010, A\&A, 517, A32

Piatti, A. E., Clariá, J. J., \& Bica, E. 1998, ApJS, 116, 263

Piatti, A. E., Clariá, J. J., \& Bica, E. 2000, A\&A, 360, 529
Platais, I., Kozhurina-Platais, V., Barnes, S., et al. 2001, AJ, 122, 1486

Pöhnl, H., \& Paunzen, E. 2010, A\&A, 514, A81

Przybilla, N., Nieva, M.-F., \& Butler, K. 2008, ApJ, 688, L103

Santos, N. C., Lovis, C., Melendez, J., et al. 2012, A\&A, 538, A151

Schlegel, D. J., Finkbeiner, D. P., \& Davis, M. 1998, ApJ, 500, 525

Schuler, S. C., Plunkett, A. L., King, J. R., \& Pinsonneault, M. H. 2010, PASP, 122,766

Sestito, P., Randich, S., Mermilliod, J.-C., \& Pallavicini, R. 2003, A\&A, 407, 289

Simón-Díaz, S. 2010, A\&A, 510, A22

Skrutskie, M. F., Cutri, R. M., Stiening, R., et al. 2006, AJ, 131, 1163

Stetson, P. B. 2000, PASP, 112, 925

Subramaniam, A., Carraro, G., \& Janes, K. A. 2010, MNRAS, 404, 1385

Sung, H., Bessell, M. S., Lee, H.-W., Kang, Y. H., \& Lee, S.-W. 1999, MNRAS, 310,982

van Leeuwen, F. 2009, A\&A, 497, 209

Villanova, S., Carraro, G., \& Saviane, I. 2009, A\&A, 504, 845 

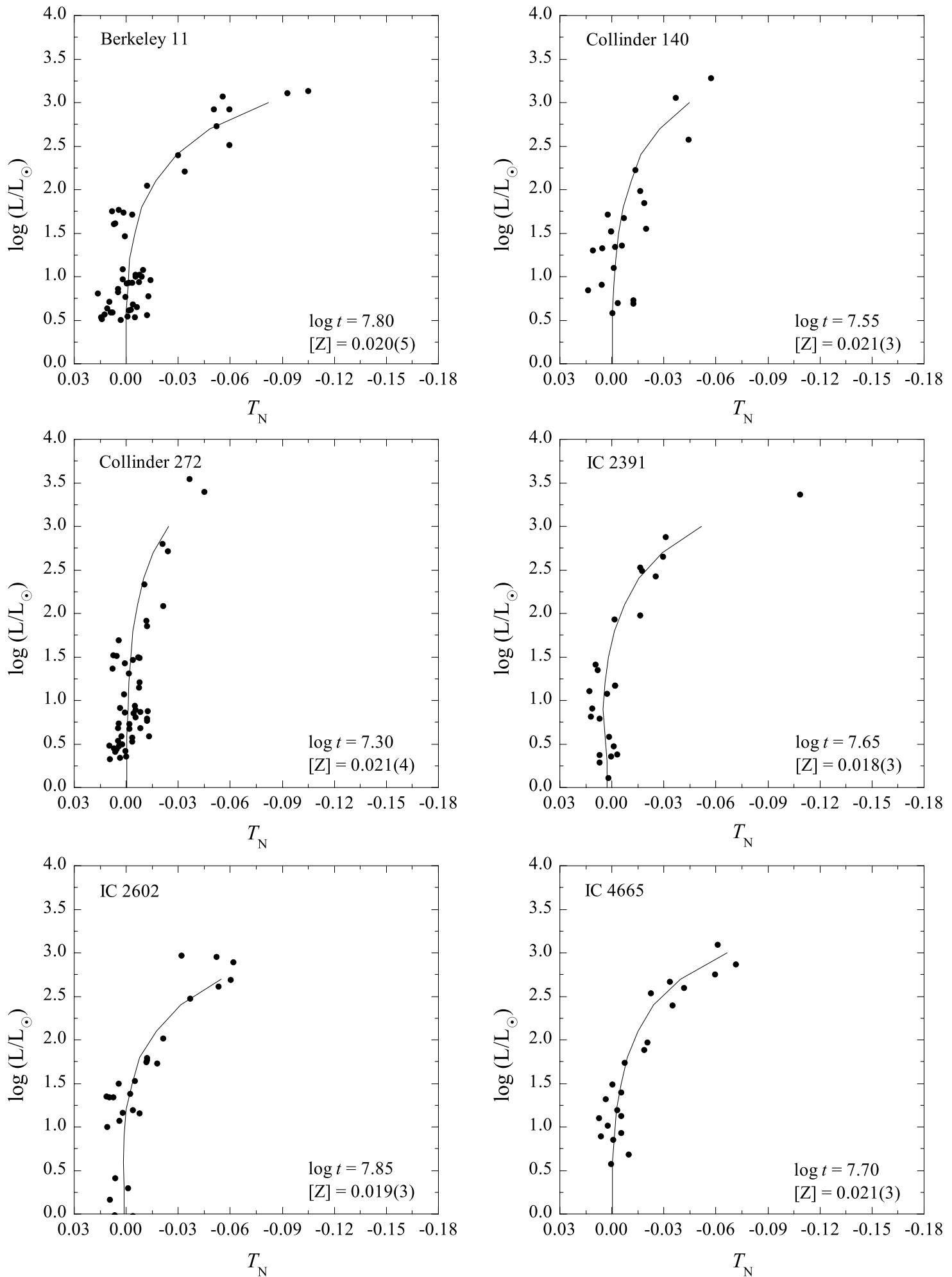

Fig. 7. Best fits of the differential grids by Pöhnl \& Paunzen (2010) to data from the programme open clusters, in order to determine the fundamental parameters including metallicity. For clarity, $T_{\mathrm{N}}$ are effective temperatures normalised to the ZAMS. An equal scale is used for a better comparison of different ages and metallicities. Owing to interpolation in the available grids, the most luminous stars are sometimes not covered by the isochrone; however, for older clusters we also included evolved stars to illustrate the continuation of the cluster sequences. 
M. Netopil and E. Paunzen: Towards a photometric metallicity scale for open clusters


Fig. 7. continued. 
A\&A 557, A10 (2013)
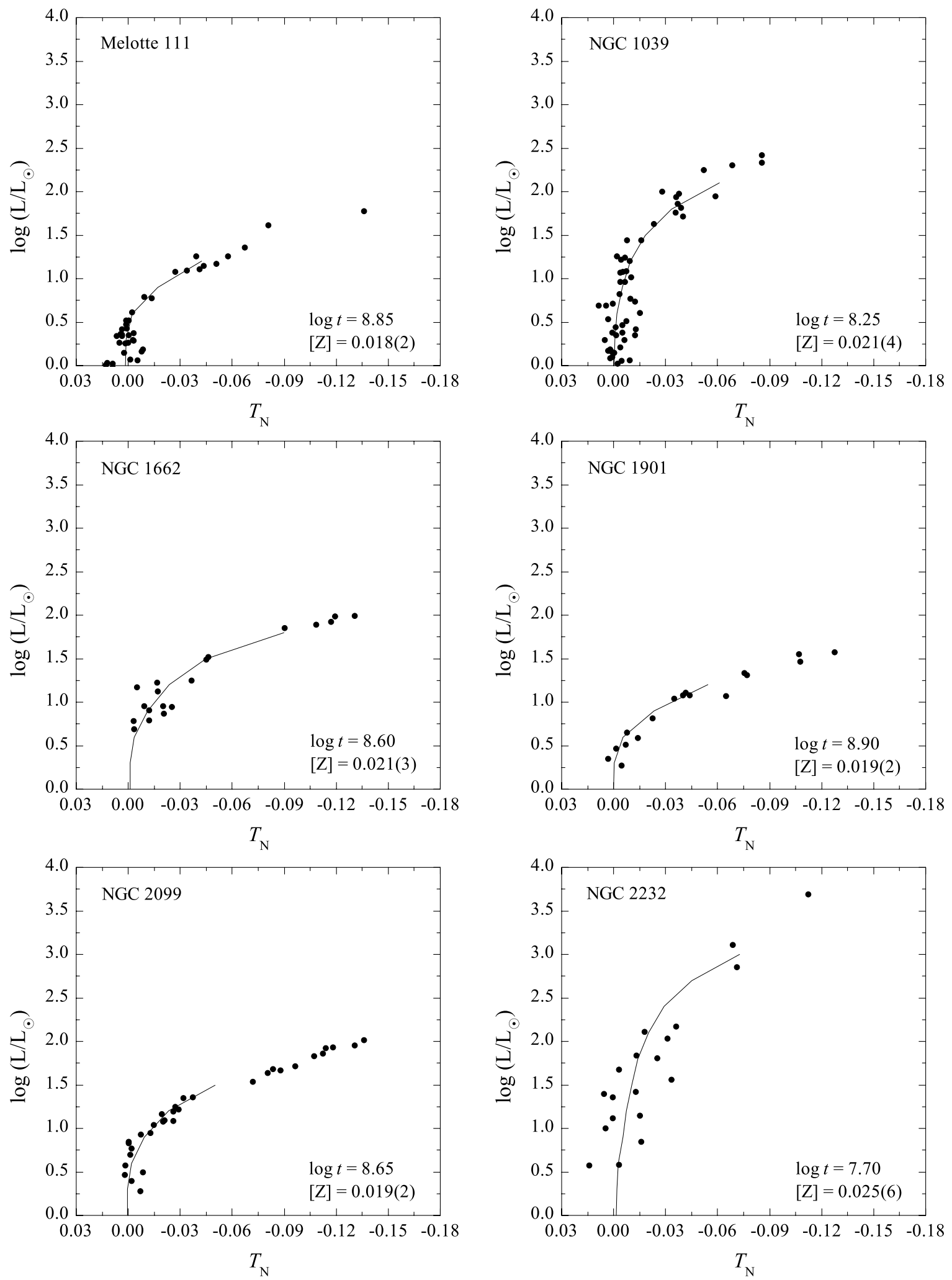

Fig. 7. continued. 
M. Netopil and E. Paunzen: Towards a photometric metallicity scale for open clusters
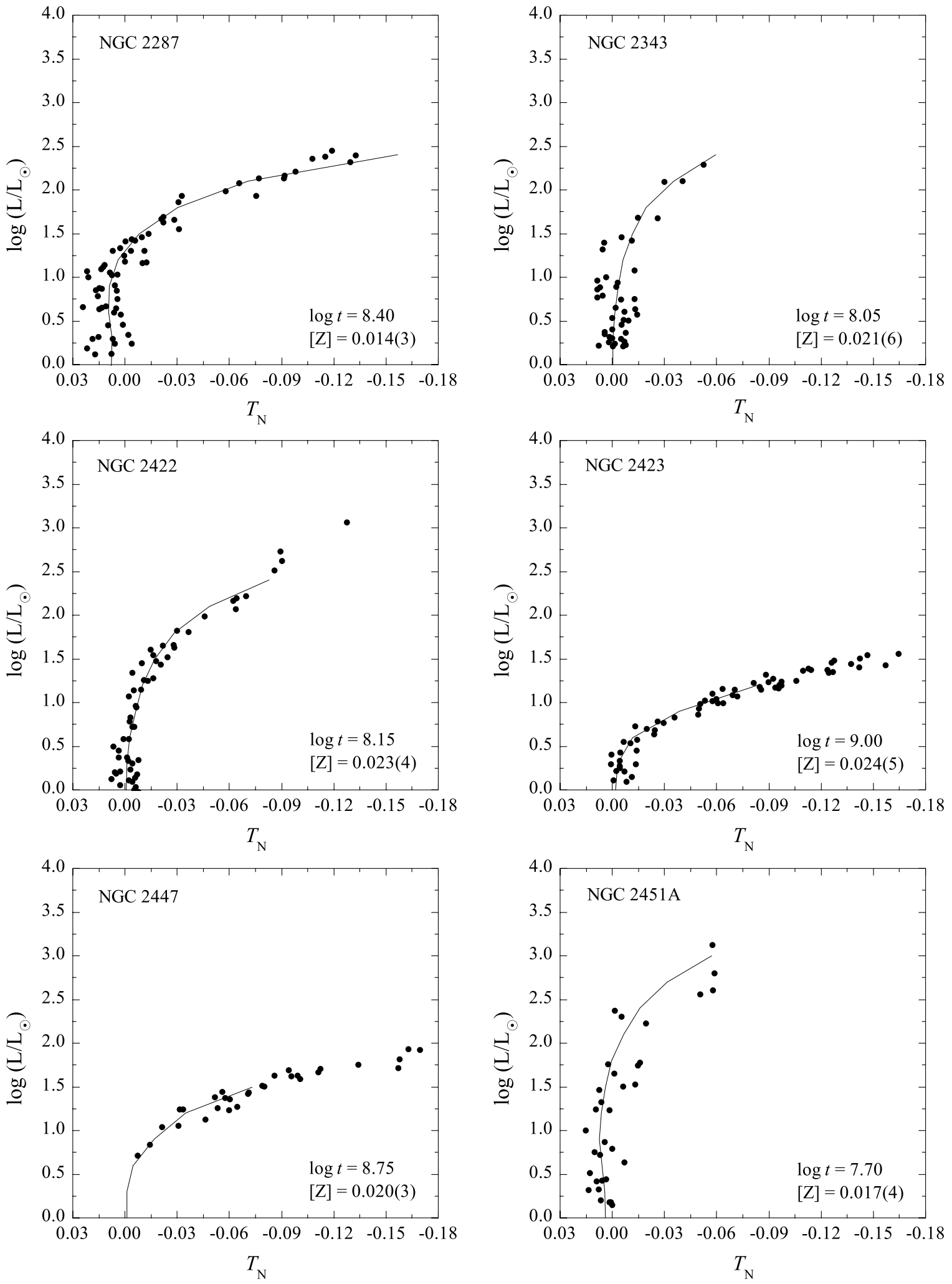

Fig. 7. continued. 

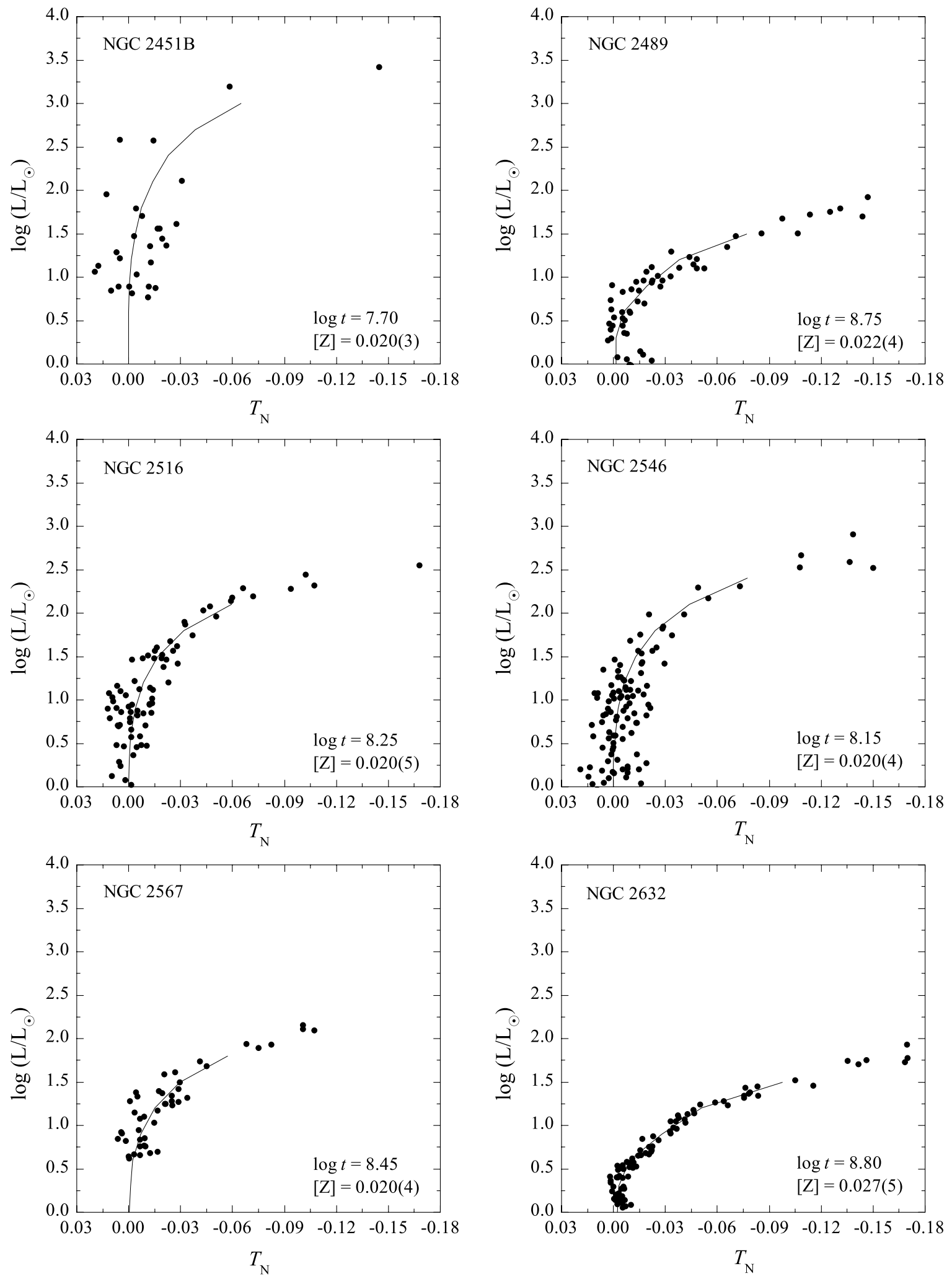

Fig. 7. continued. 
M. Netopil and E. Paunzen: Towards a photometric metallicity scale for open clusters
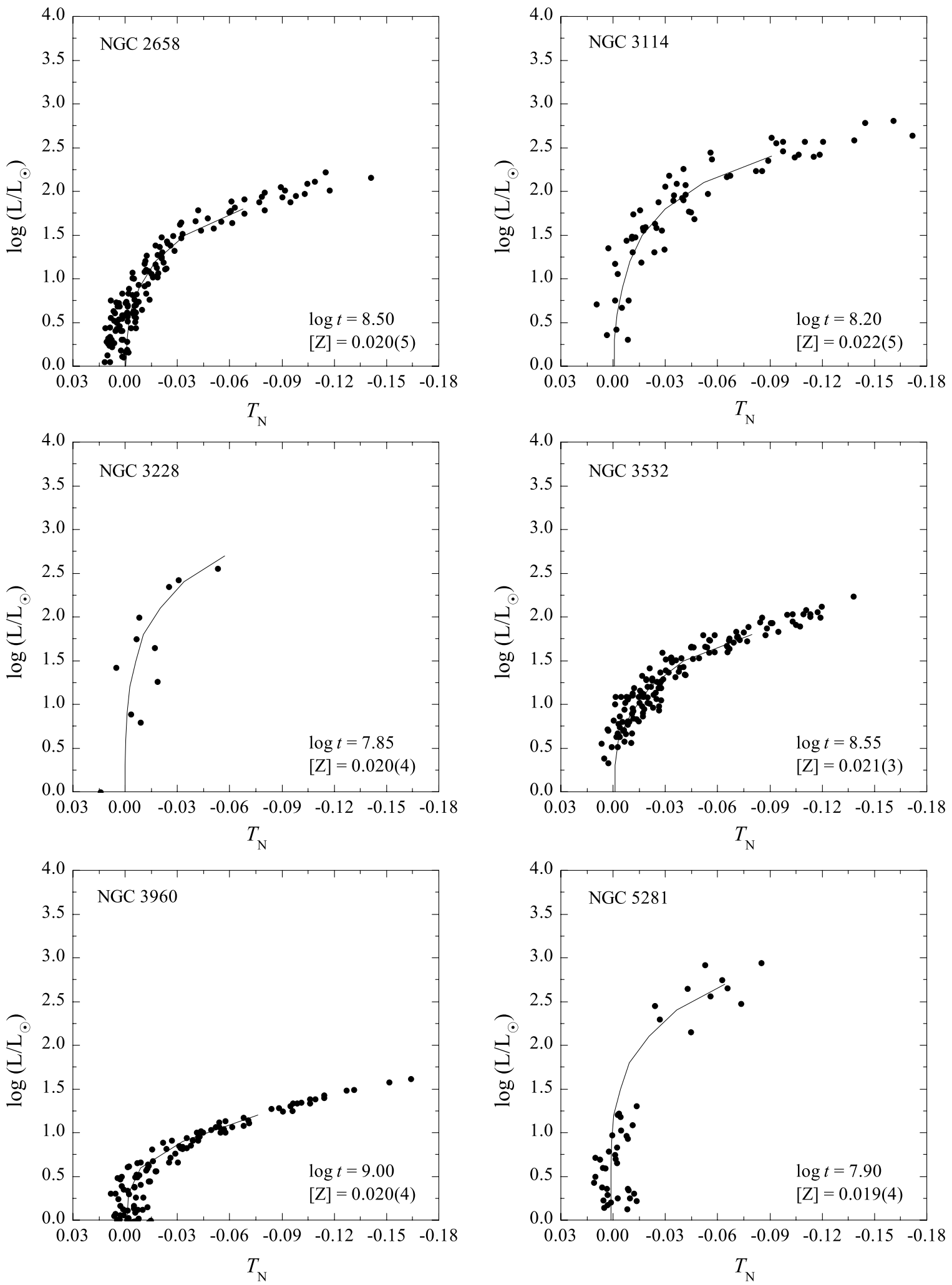

Fig. 7. continued. 
A\&A 557, A10 (2013)
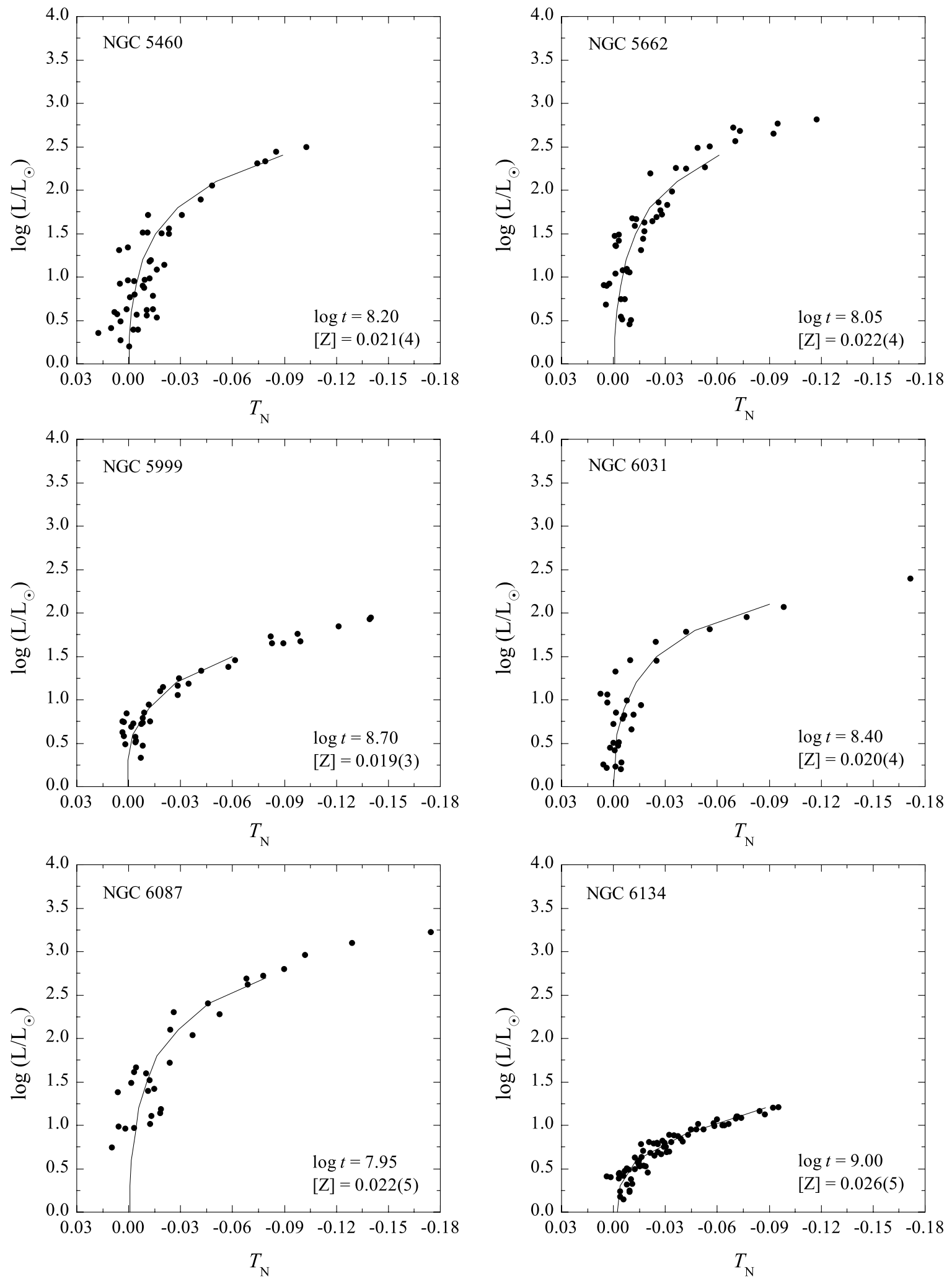

Fig. 7. continued. 
M. Netopil and E. Paunzen: Towards a photometric metallicity scale for open clusters
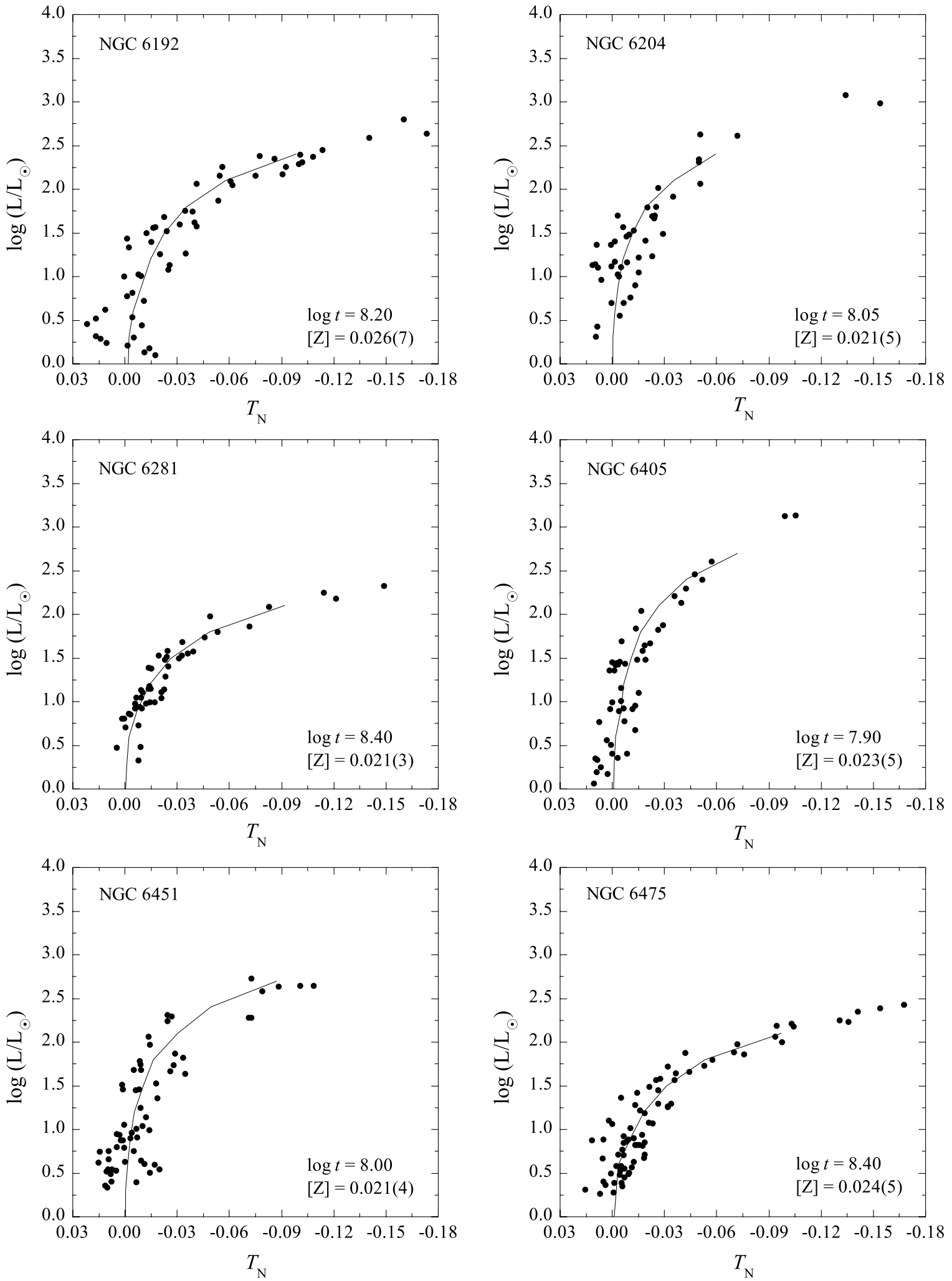

Fig. 7. continued. 
A\&A 557, A10 (2013)
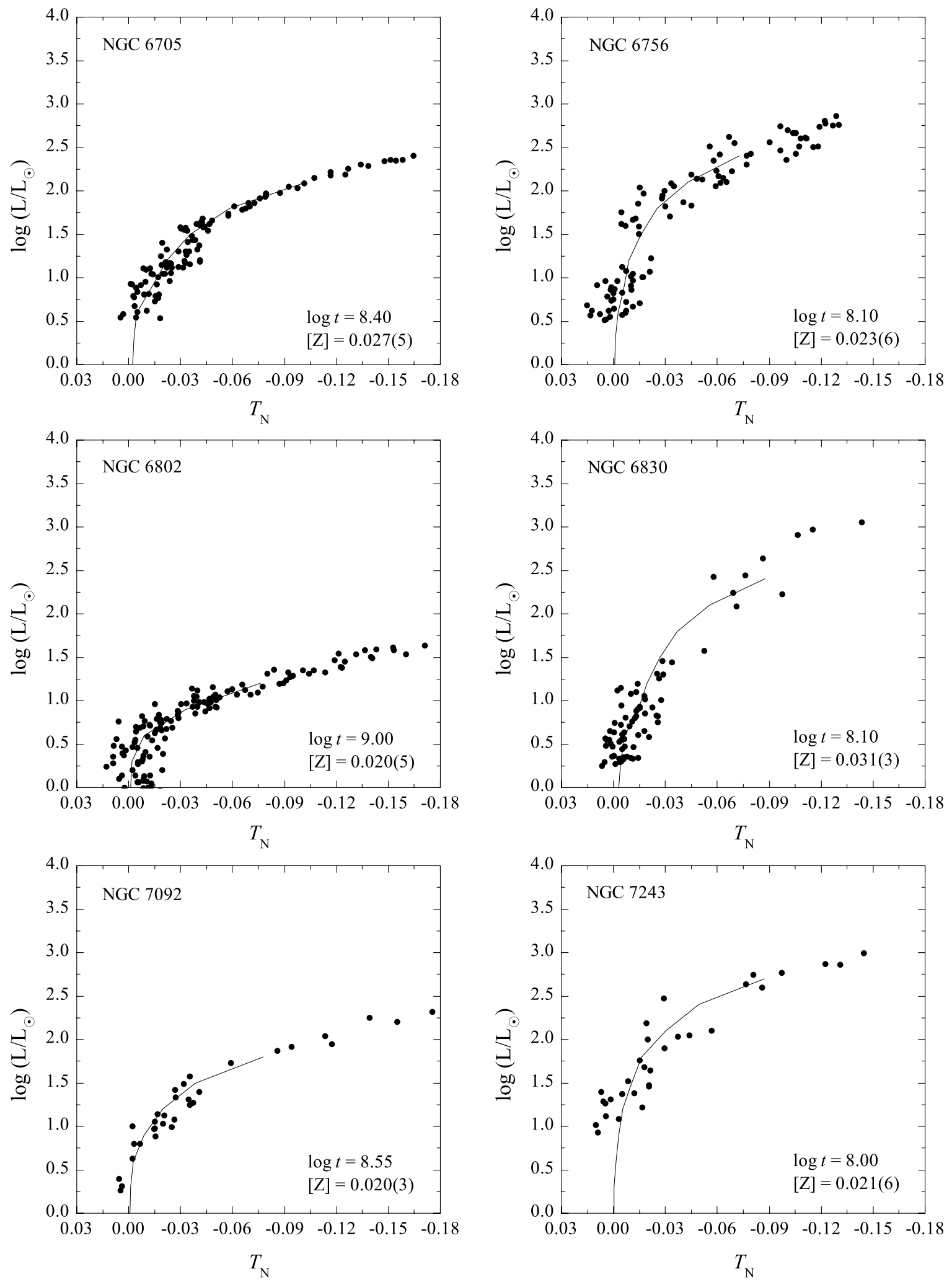

Fig. 7. continued. 
M. Netopil and E. Paunzen: Towards a photometric metallicity scale for open clusters
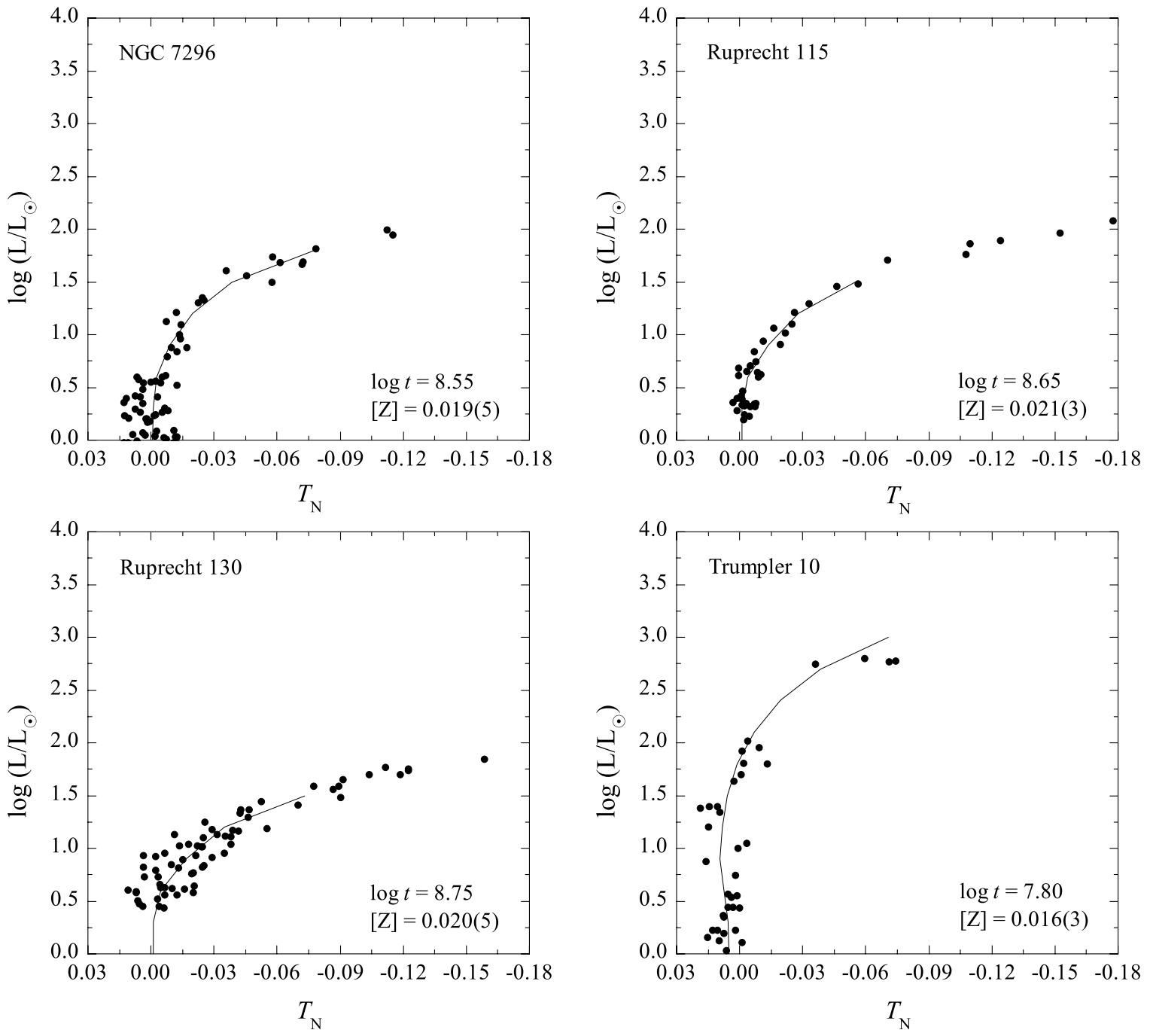

Fig. 7. continued. 